\title{
INSTABILITIES IN THE NON-UNIFORMLY ROTATING MEDIUM WITH TEMPERATURE STRATIFICATION IN THE EXTERNAL UNIFORM MAGNETIC FIELD
}

\author{
(D) Michael Kopp ${ }^{1}$, (D)Anatoly Tur ${ }^{3}$, (DVolodymyr Yanovsky ${ }^{1,2 *}$ \\ ${ }^{I}$ Institute for Single Cristals, Nat. Academy of Science Ukraine, \\ Nauky Ave. 60, Kharkov 31001,Ukraine \\ ${ }^{2}$ V.N. Karazin Kharkiv National University \\ 4, Svoboda Sq., Kharkiv, 61022, Ukraine \\ ${ }^{3}$ Universite Toulouse [UPS], CNRS, Institute of Research for Astrophysics and Planetology \\ 9 avenue du Colonel Roche, BP 44346, 31028 Toulouse Cedex 4, France \\ *E-mail:yanovsky@isc.kharkov.ua \\ Received December 20, 2018; revised February 14, 2019; accepted February 21, 2019
}

In this paper the stability of the non-uniformly rotating cylindrical plasma in the axial uniform magnetic field with the vertical temperature gradient is investigated. In the approximation of geometrical optics a dispersion equation for small axisymmetric perturbations is obtained with the effects of viscosity, ohmic and heat conductive dissipation taken into account. The stability criteria for azimuthal plasma flows are obtained in the presence of the vertical temperature gradient and the constant magnetic field. The Rayleigh-Benard problem for stationary convection in the non-uniformly rotating layer of the electrically conducting fluid in the axial uniform magnetic field is considered. In the linear theory of stationary convection the critical value of the Rayleigh number $R a_{c}$ subject to the profile of the inhomogeneous rotation (Rossby number $R o$ ) is obtained. It is shown that the negative values of the Rossby number $R o<0$ have a destabilizing effect, since the critical Rayleigh number $R a_{c}$ becomes smaller, than in the case of the uniform rotation $R o=0$, or of the rotation with positive Rossby numbers $R o>0$. To describe the nonlinear convective phenomena the local Cartesian coordinate system was used, where the inhomogeneous rotation of the fluid layer was represented as the rotation with a constant angular velocity $\vec{\Omega}_{0}$ and azimuthal shear $\vec{U}_{0}(x)$ with linear dependence on the coordinate $x$. As a result of applying the method of perturbation theory for the small parameter of supercriticality of the stationary Rayleigh number a nonlinear Ginzburg-Landau equation was obtaned. This equation describes the evolution of the finite amplitude of perturbations by utilizing the solution of the Ginzburg-Landau equation. It is shown that the weakly nonlinear convection based on the equations of the six-mode $(6 D)$ Lorentz model transforms into the identical Ginzburg-Landau equation. By utilizing the solution of the GinzburgLandau equation, we determined the dynamics of unsteady heat transfer for various profiles of the angular velocity of the rotation of electrically conductive fluid.

KEY WORDS: magnetorotational instability, Rayleigh-Benard convection, nonlinear theory, Ginzburg-Landau equation

\section{НЕСТІЙКОСТІ В СЕРЕДОВИЩІ, ЯКЕ НЕОДНОРІДНО ОБЕРТАЕТЬСЯ 3 ТЕМПЕРАТУРНОЮ СТРАТИФІКАЦІЕЮ У ЗОВНІШНЬОМУ ОДНОРІДНОМУ МАГНІТНОМУ ПОЛІ Михайло Й. Копп ${ }^{1}$, Анатолій В. Тур ${ }^{3}$, Володимир В. Яновський 1,2 \\ ${ }^{1}$ Інститут монокристалів, Національна Академія Наук Украйни пр. Науки 60, 61001 Харків, Україна \\ ${ }^{2}$ Харківський національний університет імені В.Н. Каразина майдан Свободи, 4, 61022, Харків, Украӥна \\ ${ }^{3}$ Universite Toulouse [UPS], CNRS, Institute of Research for Astrophysics and Planetology \\ 9 avenue du Colonel Roche, BP 44346, 31028 Toulouse Cedex 4, France}

Досліджується стійкість циліндричної плазми, що неоднорідно обертається в аксіальному однорідному магнітному полі 3 вертикальним градієнтом температури. У наближенні геометричної оптики отримано дисперсійне рівняння для малих осесиметричних збурень з урахуванням ефектів в'язкості, омічної та теплопровідної дисипації. Знайдено критерії стійкості азимутальних течій плазми при наявності вертикального градієнта температури і постійного магнітного поля. Розглянуто задачу Релея-Бенара для стаціонарної конвекції в шарі електропровідної рідини, що неоднорідно обертається в аксіальному магнітному полі. У лінійній теорії стаціонарної конвекції отримано критичне значення числа Релея $R a_{c}$ в залежності від профілю неоднорідного обертання (числа Росбі $R o$ ). Показано, що негативні значення числа Росбі $R o<0$ надають дестабілізуючий ефект, оскільки критичне число Релея $R a_{c}$ стає меншим, ніж у разі однорідного обертання $R o=0$ або обертання $з$ позитивними числами Росбі $R o>0$. Для опису нелінійних конвективних явищ використовувалася локальна декартова система координат, в якій неоднорідне обертання шару рідини представляється у вигляді обертання 3 постійною кутовою швидкістю $\vec{\Omega}_{0}$ і азимутальним широм $\vec{U}_{0}(x)$, профіль швидкості якого є локально лінійним. В результаті застосування методу теорії збурень за малим параметром надкритичності стаціонарного числа Релея отримано нелінійне рівняння типу Гінзбурга-Ландау, що описує еволюцію кінцевої амплітуди збурень. Показано, що розглянута слабонелінійна 
конвекція на основі рівнянь шести-модової (6D) моделі Лоренца перетворюється в ідентичне рівняння Гінзбурга-Ландау. Використовуючи рішення рівняння Гінзбурга-Ландау, ми визначили динаміку нестаціонарного переносу тепла для різних профілів кутової швидкості обертання електропровідної рідини.

КЛЮЧОВІ СЛОВА: магнітообертальна нестійкість, конвекція Релея-Бенара, слабонелінійна теорія, рівняння ГінзбургаЛандау

\section{НЕУСТОЙЧИВОСТИ В НЕОДНОРОДНО ВРАЩАЮЩЕЙСЯ СРЕДЕ С ТЕМПЕРАТУРНОЙ СТРАТИФИКАЦИЕЙ ВО ВНЕШНЕМ ОДНОРОДНОМ МАГНИТНОМ ПОЛЕ \\ Михаил И. Копп ${ }^{1}$, Анатолий В. Тур ${ }^{3}$, Владимир В. Яновский ${ }^{1,2}$ \\ ${ }^{1}$ Институт монокристаллов, Наџиональная Академия Наук Украины пр. Науки 60, 61001 Харьков, Украина \\ ${ }^{2}$ Харьковский национальный университет имени В.Н. Каразина пл. Свободы, 4, 61022, Харьков, Украина \\ ${ }^{3}$ Universite Toulouse [UPS], CNRS, Institute of Research for Astrophysics and Planetology \\ 9 avenue du Colonel Roche, BP 44346, 31028 Toulouse Cedex 4, France}

Исследуется устойчивость неоднородно вращающейся цилиндрической плазмы в аксиальном однородном магнитном поле с вертикальным градиентом температуры. В приближении геометрической оптики получено дисперсионное уравнение для малых осесимметричных возмущений с учетом эффектов вязкости, омической и теплопроводной диссипации. Найдены критерии устойчивости азимутальных течений плазмы при наличии вертикального градиента температуры и постоянного магнитного поля. Рассмотрена задача Рэлея-Бенара для стационарной конвекции в неоднородно вращающемся слое электропроводящей жидкости в аксиальном однородном магнитном поле. В линейной теории стационарной конвекции получено критическое значение числа Рэлея $R a_{c}$ в зависимости от профиля неоднородного вращения (числа Россби $R o$ ). Показано, что отрицательные значения числа Россби $R o<0$ оказывают дестабилизирующий эффект, так как критическое число Рэлея $R a_{c}$ становится меньше, чем в случае однородного вращения $R o=0$ или вращения с положительными числами Россби $R o>0$. Для описания нелинейных конвективных явлений использовалась локальная декартовая система координат, в которой неоднородное вращение слоя жидкости представляется в виде вращения с постоянной угловой скоростью $\vec{\Omega}_{0}$ и азимутальным широм $\vec{U}_{0}(x)$, профиль скорости которого локально линеен. В результате применения метода теории возмущений по малому параметру надкритичности стационарного числа Рэлея. получено нелинейное уравнение типа Гинзбурга-Ландау, описывающее эволюцию конечной амплитуды возмущений. Показано, что рассматриваемая слабонелинейная конвекция на основе уравнений шести-модовой модели Лоренца преобразуется в идентичное уравнение Гинзбурга-Ландау. Используя решение уравнения Гинзбурга-Ландау, мы определили динамику нестационарного переноса тепла для различных профилей угловой скорости вращения электропроводящей жидкости.

КЛЮЧЕВЫЕ СЛОВА: магнитовращательная неустойчивость, конвекция Рэлея-Бенара, слабонелинейная теория, уравнение Гинзбурга-Ландау

Fluid flow caused by a temperature gradient in the gravitational field, known as the phenomenon of free convection [1-3], plays an important role both in natural phenomena and in engineering and industrial applications. For several decades, free convection in liquid layers or the Rayleigh-Benard convection has been theoretically and experimentally investigated. Of particular interest are the problems related to the effect of rotation and magnetic field on the Rayleigh-Benard convection, for example, because of their applications to the theory of vortex and magnetic dynamo [4-6]. Convection, in which the axis of rotation of a medium and that of the uniform magnetic field coincide with the direction of the gravity vector, was well studied in [1-2]. The case when the directions of the axes of rotation and that of magnetic field are perpendicular to each other, and perpendicular to the direction of the gravity vector, is also of interest for solving some astrophysical problems. Such a formulation of the problem corresponds to the convection in the fluid layers located in the equatorial region of a rotating object, where the azimuthal magnetic field plays a significant role. The linear theory of such convection was first formulated in [7-8]. The linear theory of rotating magnetic convection for an arbitrary deviation of the axes of rotation and that of the magnetic field from the vertical axis (field of gravity) was developed in [9]. The studies listed above constitute a linear theory that provides information on the convection onset. It is obvious that linear models do not provide information on the final amplitude of convection. This amplitude occurs when interaction between several perturbation modes takes place. Therefore, it is important to realize the physical mechanism of nonlinear effects and to quantify the heat and mass transfer in terms of finite amplitudes. Up to date, there is no rigorous nonlinear model that can be solved analytically. Currently, to construct a nonlinear theory of convection, the perturbation method developed in [10] is widely used. This work shows that the initial heat transfer by convection depends linearly on the Rayleigh number, and then, at higher Rayleigh numbers, the heat transfer is slightly different from the linear case. The authors of [10] called this process weakly nonlinear, where the nonlinearity depended on the linear case. The weakly nonlinear theory of convection was further developed with regard to modulation of the parameters that control the convection process, what is very important for solving many technological problems. Different types of modulation, such as rotation [11-14], gravity [15-17], temperature [18-20] and magnetic field [21-22], were studied for stationary weakly nonlinear convection in various media: porous media, nanofluids, and so on. In these papers [11-22] the effect of modulation of the parameters (rotation, gravity, temperature, magnetic field) on the heat and mass transfer in convective media was determined. Despite the enormous amount of works on the Rayleigh-Benard convection, there is still a certain gap in the study of the 
influence of inhomogeneous (or differential) rotation on convective processes. The interest to these studies is primarily caused by various astrophysical problems. It is known that the majority of various space objects consisting of dense gases or liquid (Jupiter, Saturn, Sun, etc.) rotate non-uniformly, i.e. different parts of the object rotate around a common axis of rotation with different angular velocities. Differential rotation is also observed in galaxies, accretion disks, and extended rings of planets. Besides, such large-scale vortex structures as typhoons, cyclones and anticyclones, etc. also rotate non-uniformly.

The stability of the inhomogeneous rotation of the ideally conducting medium in the magnetic field was first considered in [23-24]. These works also show that a weak axial magnetic field destabilizes the azimuthal differential rotation of plasma, and when the condition $d \Omega^{2} / d R<0$ is satisfied, a magneto-rotational instability (MRI) or standard MRI (SMRI) in the non-dissipative plasma occurs. Since this condition is also satisfied for Keplerian flows $\Omega \sim R^{-3 / 2}$, the MRI is the most likely source of turbulence in the accretion disks. The MRI discovery stimulated numerous theoretical studies. The first theoretical studies that dealt with the problem of accretion flows were carried out in the approximation of a non-dissipative plasma with the radial thermal stratification [25] and the magnetization of the heat fluxes [26] taken into account. In [27] the stability of the differentially rotating plasma in the axial magnetic field was studied with simultaneous consideration of both dissipative effects (viscosity and Ohmic dissipation) and thermal radial stratification of plasma. MRI in a spiral magnetic field, i.e. with nontrivial topology was studied in [28-29]. While studying the MRI, the differential rotation of the medium is simulated by the Couette flow between two cylinders rotating at different angular velocities (Fig. 1a), which is convenient for carrying out laboratory experiments [30].

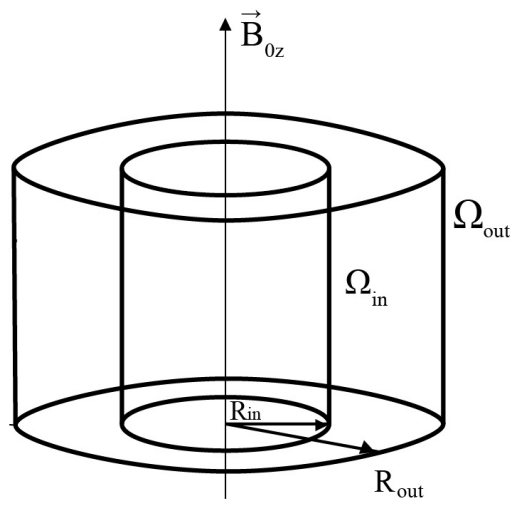

a)

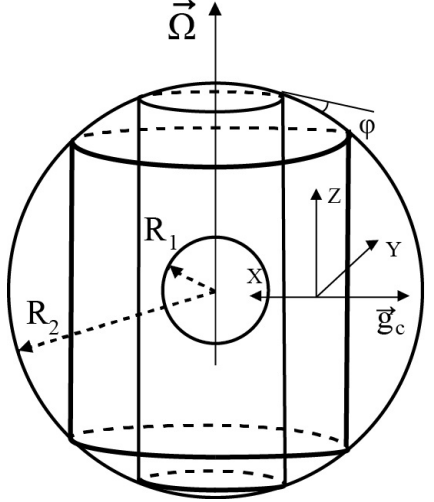

б)

Fig.1. a) geometry of the problem for standard MRI: two concentric cylinders with radii $R_{\text {in }}=R_{1}$ and $R_{\text {out }}=R_{2}$ rotating with velocities $\Omega_{\text {in }}=\Omega_{1}$ and $\Omega_{\text {out }}=\Omega_{2}$. $B_{0 z}$ - axial magnetic field directed vertically upwards; b) Convective Busse dynamo model for a layer of the electrically conductive fluid in the rotating magneto-convection.

In [31] various models of thermal convection in rapidly rotating fluids penetrated by strong magnetic fields are discussed. A special attention is paid to the probability, that the magnetic field can be supported by the dynamo action, but not by the electric currents applied externally. In [31] an overview of two dynamo models is given. This is the Childress-Soward flat layer model [32] and the annulus model by Busse [33]. The Childress-Soward model operates in the convective flat layers of fluid located in moderate and subpolar latitudes (Fig. 2a) of the space object.

For the terrestrial dynamo, the Busse model operates in the equatorial layers, where the azimuth magnetic field plays a significant role. The electrically conductive fluid rotates in the annular region located between the solid core and the Earth crust. The theory of this process was developed in [33-35], where the model of rotating cylinders was used. According to this theory [35] the outer cylinder rotates at a constant angular velocity $\Omega_{2}$, while the inner one remains stationary $\Omega_{1}=0$ (Fig. 1b). Convective flows (Benard cells) occur in the fluid layer between the cylinders due to the temperature difference between the inner $T_{\text {in }}$ and outer $T_{\text {out }}$ cylinders $T_{\text {out }}>T_{\text {in }}$. The difference in the heights of the inner $h_{1}$ and outer $h_{2}$ cylinders leads to a similar effect of the Coriolis force on $\beta$-plane.

These models do not completely solve the problems of geodynamo, for example, the problem of magnetic field inversions. Unlike the Childress-Soward and Busse models, the stability of a non-uniformly rotating layer of the electrically conducting fluid in the axial magnetic field, in which the lower surface of the layer $\left(T_{d}\right)$ is hotter than the upper one $\left(T_{u}\right)$ (Rayleigh-Benard problem): $T_{d}>T_{u}$, was studied in [36] (Fig. 2b). In [36] a chaotic regime based on the nonlinear dynamics equations of a six-dimensional phase space was studied. The analysis of these equations revealed the existence of a complex chaotic structure - a strange attractor. Besides, a convection mode was determined, in which some chaotic change in direction (inversion) and amplitude of the perturbed magnetic field occurs, with the the medium inhomogeneous rotation taken into account. 


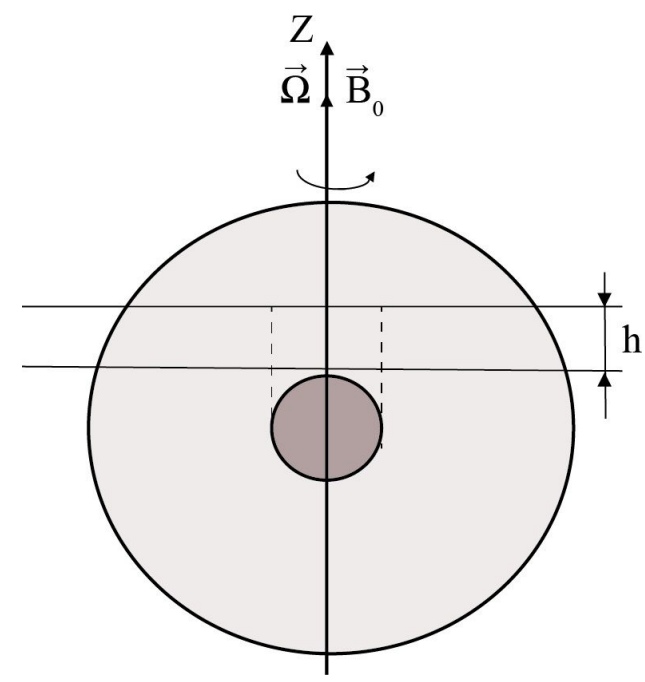

a)

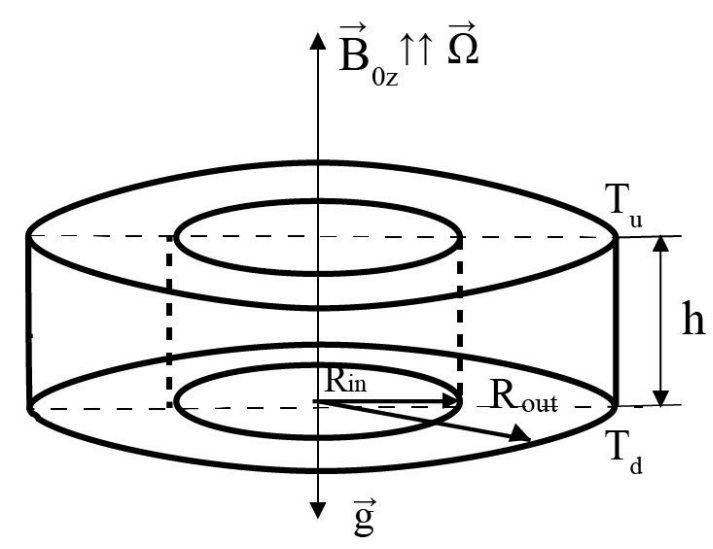

б)

Fig.2. a) A layer (thickness $h$ ) of the electrically conductive fluid of the astrophysical object that rotates at a non-uniform velocity $\vec{\Omega}(R)$ in the axial magnetic field $\vec{B}_{0}$ (analogous to the Childress-Soward dynamo model); b) geometric description of the convective dynamo simulated by Couette-Taylor flow in a thin layer of the electrically conducting fluid.

The aim of this work is to study local instabilities in rotating flows in the presence of a constant vertical magnetic field and the temperature gradient in the field of gravity, as well as the development of a linear and weakly nonlinear theory of the stationary convection in a non-uniformly rotating layer of the electrically conductive fluid in the axial uniform magnetic field.

The results obtained in this work can be applied to various astrophysical and geophysical problems, which consider magnetic convection in rotating layers of the Earth interior, the Sun, hot galactic clusters, accretion disks and other objects.

\section{LOCAL INSTABILITIES IN A MAGNETIZED ROTATING FLOW WITH WEAK TEMPERATURE STRATIFICATION \\ Basic equations of small perturbations evolution}

Let us consider the flow dynamics of a non-uniformly rotating conductive fluid (plasma) in a constant gravitational $g$ and magnetic $B_{0}$ fields with a constant vertical temperature gradient $\nabla T_{0}=c o n s t=-A e$, where $A>0$ is a constant gradient, $e$ is a unit vector directed vertically upwards along $Z$ axis. The stationary flow of the non-homogeneously rotating fluid will be simulated by the Couette-Taylor flow, located between two rotating cylinders with the angular velocity of rotation $\Omega(R)$ :

$$
\Omega(R)=\frac{\Omega_{2} R_{2}^{2}-\Omega_{1} R_{1}^{2}}{R_{2}^{2}-R_{1}^{2}}+\frac{\left(\Omega_{1}-\Omega_{2}\right) R_{1}^{2} R_{2}^{2}}{R^{2}\left(R_{2}^{2}-R_{1}^{2}\right)},
$$

where $R_{1}=R_{\text {in }}, R_{2}=R_{\text {out }}, \Omega_{1}=\Omega_{\text {in }}, \Omega_{2}=\Omega_{\text {out }}$ - radius and angular velocity of rotation of the inner and outer cylinders, respectively.

To describe the motion of a viscous incompressible electrically conducting fluid we use the equations of magnetic hydrodynamics in the Boussinesq approximation [1-2]:

$$
\begin{gathered}
\frac{\partial \vec{v}}{\partial t}+(\vec{v} \nabla) \vec{v}=-\frac{1}{\rho_{0}} \nabla\left(P+\frac{B^{2}}{8 \pi}\right)+\frac{1}{4 \pi \rho_{0}}(\vec{B} \nabla) \vec{B}+\vec{e} g \beta T+v \nabla^{2} \vec{v} \\
\frac{\partial \vec{B}}{\partial t}+(\vec{v} \nabla) \vec{B}-(\vec{B} \nabla) \vec{v}=\eta \nabla^{2} \vec{B} \\
\frac{\partial T}{\partial t}+(\vec{v} \nabla) T=\chi \nabla^{2} T \\
\operatorname{div} \vec{B}=0, \quad \operatorname{div} \vec{v}=0
\end{gathered}
$$


where $\beta$ is the coefficient of thermal expansion, $\rho_{0}=$ const is the density of the medium, $v$ is the coefficient of kinematic viscosity, $\eta=c^{2} / 4 \pi \sigma$ is the coefficient of magnetic viscosity, $\sigma$ is the coefficient of electrical conductivity, $\chi$ is the coefficient of the medium thermal conductivity.

We assume that the uniform (constant) magnetic field $\vec{B}_{0}$ is directed along the axis $O Z$. The field will be further called axial in the cylindrical coordinate system $(R, \phi, z)$. The direction of the magnetic field coincides with the axis of rotation of the fluid $\vec{\Omega} \| O Z$, which rotates in the azimuthal direction with velocity $\vec{v}_{0}=R \Omega(R) \vec{e}_{\phi}$. The stationary state of the system satisfies the following equations:

$$
\Omega^{2} R=\frac{1}{\rho_{0}} \frac{d p_{0}}{d R}, \frac{1}{\rho_{0}} \frac{d p_{0}}{d z}=-g \beta T_{0}, \frac{d^{2} T_{0}}{d z^{2}}=0 .
$$

Equation (5) shows that the centrifugal equilibrium is established in the radial direction, and the hydrostatic one in the vertical direction.

Our main task is to consider the problem of the stability of small perturbations of physical quantities $(\vec{u}, \vec{b}, p, \theta)$ against a background of the stationary state (5). By representing all the quantities in equations (1)-(4) as the sum of the stationary and perturbed parts $\vec{v}=\vec{v}_{0}+\vec{u}, \vec{B}=\vec{B}_{0}+\vec{b}, \quad P=p_{0}+p, T=T_{0}+\theta$, we obtain the evolution equations for small perturbations in the linear approximation:

$$
\left(\begin{array}{ccc}
\partial_{t}-\nu \nabla^{2}+M_{\Omega}+\vec{v}_{0} \cdot \nabla & -\frac{\vec{B}_{0} \cdot \nabla}{4 \pi \rho_{0}} & \vec{e} g \beta \\
-\vec{B}_{0} \cdot \nabla & \partial_{t}-\eta \nabla^{2}-M_{\Omega}+\vec{v}_{0} \cdot \nabla & 0 \\
-A \cdot \vec{e} & 0 & \partial_{t}-\chi \nabla^{2}+\vec{v}_{0} \cdot \nabla
\end{array}\right) \cdot\left(\begin{array}{c}
\vec{u} \\
\vec{b} \\
\theta
\end{array}\right)=-\frac{\nabla}{\rho_{0}}\left(\begin{array}{c}
p+\frac{\vec{B}_{0} \cdot b}{4 \pi} \\
0 \\
0
\end{array}\right),
$$

where indication $M_{\Omega}$ for the matrix of the non-uniform rotation is entered [28]:

$$
M_{\Omega}=\left(\begin{array}{ccc}
0 & -\Omega & 0 \\
\Omega+R \frac{d \Omega}{d R} & 0 & 0 \\
0 & 0 & 0
\end{array}\right) .
$$

Perturbed fields $\vec{u}, \vec{b}$ also satisfy the solenoid condition:

$$
\operatorname{div} \vec{b}=0, \quad \operatorname{div} \vec{u}=0 .
$$

As long as the medium is stratified by temperature and rotates with an inhomogeneous angular velocity, a justification for the applicability of the limit of geometric optics on the bases of the asymptotic WKB (Wentzel - Kramers Brillouin) method [37] should be provided.

\section{Asymptotic WKB method and geometric optics approximation}

Let us consider the limit of the medium weak stratification when the spatial scale of the medium heterogeneity $L=\left(\frac{1}{T_{0}} \frac{d T_{0}}{d z}\right)^{-1}$ far exceeds the typical perturbation scale (wavelength) $\lambda=\frac{2 \pi}{|k|}: L \gg \lambda$. In the short-wave limit $(L \gg \lambda)$ an approximation of geometrical optics is performed, and therefore all the perturbed quantities in equations (6-7) can be represented by the dependence of the form: $\exp (i \vec{k} \vec{r}+\gamma t)$, where $\vec{k}$ is the wave vector, $\gamma$ is the amplification (or attenuation) factor of the disturbances [38].

According to [28-29] we present a more rigorous justification of the short-wave approximation using the asymptotic WKB method. For this purpose we represent the solutions of the linearized system of equations (6) in the form of an asymptotic series in the small parameter $\mathcal{E}(0<\mathcal{E} \ll 1)$ : 


$$
\left(\begin{array}{c}
\vec{u} \\
\vec{b} \\
p \\
\theta
\end{array}\right)=e^{i \Phi(x, t) / \varepsilon}\left(\begin{array}{l}
\vec{u}^{(0)}(x, t)+\varepsilon \vec{u}^{(1)}(x, t)+\cdots \\
\vec{b}^{(0)}(x, t)+\varepsilon \vec{b}^{(1)}(x, t)+\cdots \\
p^{(0)}(x, t)+\varepsilon p^{(1)}(x, t)+\cdots \\
\theta^{(0)}(x, t)+\varepsilon \theta^{(1)}(x, t)+\cdots
\end{array}\right),
$$

where $x=(R, \phi, z)$ are the cylindrical coordinates recorded in vector form; $\Phi(x, t)$ is a scalar function, called the phase (or eikonal) of the perturbed quantities oscillations; $\vec{u}^{(n)}, \vec{b}^{(n)}, \theta^{(n)}, p^{(n)}(n=0,1, \ldots)$ are the amplitudes of disturbances. The dissipative processes, when using the asymptotic expansion (8), have an effect in the second order of $\varepsilon$ smallness [28-29], i.e. $v=\varepsilon^{2} \tilde{v}, \eta=\varepsilon^{2} \tilde{\eta}, \chi=\varepsilon^{2} \tilde{\chi}$. For convenience, we introduce the indication for the derivative along the fluid flow lines:

$$
\frac{D}{D t} \equiv \frac{\partial}{\partial t}+\vec{v}_{0} \cdot \nabla
$$

Substituting decompositions (8) into the system of equations (6), we obtain the system of local differential equations for $\mathcal{E}^{-1}$ and $\mathcal{E}^{0}$ orders:

$$
\begin{aligned}
& \varepsilon^{-1}:\left(\begin{array}{ccc}
\frac{D \Phi}{D t} & -\frac{\left(\vec{B}_{0} \cdot \nabla \Phi\right)}{4 \pi \rho_{0}} & 0 \\
-\left(\vec{B}_{0} \cdot \nabla \Phi\right) & \frac{D \Phi}{D t} & 0 \\
0 & 0 & \frac{D \Phi}{D t}
\end{array}\right) \cdot\left(\begin{array}{c}
\vec{u}^{(0)} \\
\vec{b}^{(0)}
\end{array}\right)=-\frac{\nabla \Phi}{\rho_{0}}\left(\begin{array}{c}
p^{(0)}+\frac{\vec{B}_{0} \cdot \vec{b}^{(0)}}{4 \pi} \\
0 \\
0
\end{array}\right) \\
& \varepsilon^{0}: i \cdot\left(\begin{array}{ccc}
\frac{D \Phi}{D t} & -\frac{\left(\vec{B}_{0} \cdot \nabla \Phi\right)}{4 \pi \rho_{0}} & 0 \\
-\left(\vec{B}_{0} \cdot \nabla \Phi\right) & \frac{D \Phi}{D t} & 0 \\
0 & 0 & \frac{D \Phi}{D t}
\end{array}\right) \cdot\left(\begin{array}{c}
\vec{u}^{(1)} \\
\vec{b}^{(1)} \\
\theta^{(1)}
\end{array}\right)+\frac{i \nabla \Phi}{\rho_{0}}\left(\begin{array}{c}
p^{(1)}+\frac{\vec{B}_{0} \cdot \vec{b}^{(1)}}{4 \pi} \\
0 \\
0
\end{array}\right)+ \\
& +\frac{\nabla}{\rho_{0}}\left(\begin{array}{c}
p^{(0)}+\frac{\vec{B}_{0} \cdot \vec{b}^{(0)}}{4 \pi} \\
0 \\
0
\end{array}\right)=0
\end{aligned}
$$

Solenoid conditions (7) take the form:

$$
\begin{aligned}
& \vec{u}^{(0)} \cdot \nabla \Phi=0, \quad \nabla \cdot \vec{u}^{(0)}+i \vec{u}^{(1)} \cdot \nabla \Phi=0, \\
& \vec{b}^{(0)} \cdot \nabla \Phi=0, \quad \nabla \cdot \vec{b}^{(0)}+i \vec{b}^{(1)} \cdot \nabla \Phi=0 .
\end{aligned}
$$

Next, multiplying equations (9) alternately by $\nabla \Phi, u^{(0)}, b^{(0)}$ and applying equations (11) we obtain the following relations:

$$
\frac{(\nabla \Phi)^{2}}{\rho_{0}} \cdot\left(p^{(0)}+\frac{1}{4 \pi}\left(\vec{B}_{0} \cdot \vec{b}^{(0)}\right)\right)=0, \frac{D \Phi}{D t} b^{(0)} \cdot \nabla \Phi=0, \frac{D \Phi}{D t} \theta^{(0)} \cdot \nabla \Phi=0,
$$




$$
\begin{aligned}
& \frac{D \Phi}{D t} \vec{u}^{(0)} \cdot \vec{u}^{(0)}-\frac{\left(\vec{B}_{0} \cdot \nabla \Phi\right)}{4 \pi \rho_{0}} \vec{b}^{(0)} \cdot \vec{u}^{(0)}=0, \frac{D \Phi}{D t} \vec{b}^{(0)} \cdot \vec{u}^{(0)}-\left(\vec{B}_{0} \cdot \nabla \Phi\right) \vec{u}^{(0)} \cdot \vec{u}^{(0)}=0, \frac{D \Phi}{D t} \theta^{(0)} \cdot \vec{u}^{(0)}=0, \\
& \frac{D \Phi}{D t} \vec{u}^{(0)} \cdot \vec{b}^{(0)}-\frac{\left(\vec{B}_{0} \cdot \nabla \Phi\right)}{4 \pi \rho_{0}} \vec{b}^{(0)} \cdot \vec{b}^{(0)}=0, \frac{D \Phi}{D t} \vec{b}^{(0)} \cdot \vec{b}^{(0)}-\left(\vec{B}_{0} \cdot \nabla \Phi\right) \vec{u}^{(0)} \cdot \vec{b}^{(0)}=0, \frac{D \Phi}{D t} \theta^{(0)} \cdot \vec{b}^{(0)}=0 .
\end{aligned}
$$

Due to the fact that $\nabla \Phi \neq 0, \vec{u}^{(0)} \neq 0, \vec{b}^{(0)} \neq 0, \theta^{(0)} \neq 0$ we obtain:

$$
p^{(0)}=-\frac{1}{4 \pi}\left(\vec{B}_{0} \cdot \vec{b}^{(0)}\right), \quad \frac{D \Phi}{D t}=0, \quad \vec{B}_{0} \cdot \nabla \Phi=0 .
$$

According to (12) the system of equations (10) becomes much simpler.

$$
\begin{gathered}
\left(\frac{D}{D t}+\tilde{v}(\nabla \Phi)^{2}+M_{\Omega}\right) \vec{u}^{(0)}-\frac{\left(\vec{B}_{0} \cdot \nabla\right)}{4 \pi \rho_{0}} \vec{b}^{(0)}-\vec{e} g \beta \theta^{(0)}=-\frac{i \nabla \Phi}{\rho_{0}}\left(p^{(1)}+\frac{\vec{B}_{0} \cdot \vec{b}^{(1)}}{4 \pi}\right) \\
\left(\frac{D}{D t}+\tilde{\eta}(\nabla \Phi)^{2}-M_{\Omega}\right) \vec{b}^{(0)}-\left(\vec{B}_{0} \cdot \nabla\right) \vec{u}^{(0)}=0 \\
\left(\frac{D}{D t}+\tilde{\chi}(\nabla \Phi)^{2}\right) \theta^{(0)}-A \cdot \vec{e} \cdot \vec{u}^{(0)}=0
\end{gathered}
$$

The second relation in (12) is the Hamilton-Jacobi equation with the initial condition: $\Phi(x, 0)=\Phi_{0}(x)$. Acting on this equation by operator $\nabla$ we obtain the eikonal equation:

$$
\partial_{t} \nabla \Phi+\nabla\left(\vec{v}_{0} \cdot \nabla \Phi\right)=0
$$

or

$$
\partial_{t} \nabla \Phi+e_{R}\left(\frac{\partial \Omega}{\partial R} \frac{\partial \Phi}{\partial \phi}+\Omega(R) \frac{\partial^{2} \Phi}{\partial R \partial \phi}\right)+e_{\phi} \frac{\Omega(R)}{R} \frac{\partial^{2} \Phi}{\partial \phi^{2}}+e_{z} \Omega(R) \frac{\partial^{2} \Phi}{\partial z \partial \phi}=0
$$

the initial condition for this equation is: $\nabla \Phi(x, 0)=\nabla \Phi_{0}(x)$. It is known that the phase gradient $\nabla \Phi$ is by definition a wave vector:

$$
\vec{k}=\nabla \Phi=e_{R} k_{R}+e_{\phi} k_{\phi}+e_{z} k_{z},
$$

then for the components of vector $k$ we obtain the following equations:

$$
\partial_{t} k_{R}=-\left(\frac{\partial \Omega}{\partial R} k_{\phi}+\Omega(R) R \frac{\partial k_{\phi}}{\partial \phi}\right), \partial_{t} k_{\phi}=-\Omega(R) \frac{\partial k_{\phi}}{\partial \phi}, \partial_{t} k_{z}=-\Omega(R) R \frac{\partial k_{\phi}}{\partial z} .
$$

From these equations it follows that for the axisymmetric perturbations $\left(k_{\phi}=\partial \Phi / R \partial \phi=0\right)$ the wave vectors $k_{R}$ and $k_{z}$ do not depend on time, i.e. they can be considered constant. Besides, it is known (see, for example, [37]) that geometric optics is approximated locally, where the amplitude and direction of the wave remain almost unchanged at the distance of the order of the wavelength $\lambda$, hence the wave vector $\vec{k}$ (or phase gradient $\nabla \Phi$ ) can be considered constant: $|\vec{k}|=$ const. Since the approximation of geometrical optics is well satisfied for the axisymmetric perturbations, the perturbations $\vec{u}^{(0)}, \vec{b}^{(0)}, \theta^{(0)}, p^{(1)}$ in equations (13) can be represented as plane waves:

$$
\left(\begin{array}{c}
\vec{u}^{(0)} \\
\vec{b}^{(0)} \\
\theta^{(0)} \\
p^{(1)}
\end{array}\right)=\left(\begin{array}{c}
\vec{U} \\
\vec{H} \\
\Theta \\
P
\end{array}\right) \exp \left(\gamma t+i k_{R} R+i k_{z} z\right) .
$$


After substituting (15) into the system of equations (13) we obtain the system of equations for the amplitudes of the perturbations $\vec{U}, \vec{H}, \Theta, P$ :

$$
\begin{gathered}
\left(\gamma+\omega_{v}\right) U_{R}-2 \Omega u_{\phi}=\frac{i\left(\vec{k} \vec{B}_{0}\right) H_{R}}{4 \pi \rho_{0}}-\frac{i k_{R}}{\rho_{0}} \tilde{P} \\
\left(\gamma+\omega_{v}\right) U_{\phi}+2 \Omega(1+R o) U_{R}=\frac{i\left(\vec{k} \vec{B}_{0}\right) H_{\phi}}{4 \pi \rho_{0}} \\
\left(\gamma+\omega_{v}\right) U_{z}=\frac{i\left(\vec{k} \vec{B}_{0}\right) H_{z}}{4 \pi \rho_{0}}-\frac{i k_{z}}{\rho_{0}} \tilde{P}+g \beta \Theta \\
\left(\gamma+\omega_{\eta}\right) H_{R}=i\left(\vec{k} \vec{B}_{0}\right) U_{R} \\
\left(\gamma+\omega_{\eta}\right) H_{\phi}=i\left(\vec{k} \vec{B}_{0}\right) U_{\phi}+R \frac{\partial \Omega}{\partial R} H_{R} \\
\left(\gamma+\omega_{\eta}\right) H_{z}=i\left(\vec{k} \vec{B}_{0}\right) U_{z} \\
\left(\gamma+\omega_{\chi}\right) \Theta=A U_{z} \\
k_{R} U_{R}+k_{z} U_{z}=k_{R} H_{R}+k_{z} H_{z}=0
\end{gathered}
$$

In equations (16)-(23) the indications for the total pressure $\tilde{P}=P+\frac{\vec{B}_{0} \cdot \vec{H}^{(1)}}{4 \pi}$, viscous $\omega_{v}=v|\vec{k}|^{2}$, ohmic $\omega_{\eta}=\eta|\vec{k}|^{2}$ and heat conductive $\omega_{\chi}=\chi|\vec{k}|^{2}$ dissipation frequency $\left(|\vec{k}|^{2}=k_{R}^{2}+k_{z}^{2}\right)$ are introduced, Ro is the hydrodynamic Rossby number, characterizing the heterogeneity of the medium rotation: $R o=\frac{R}{2 \Omega} \frac{\partial \Omega}{\partial R}$.

Using the method of elimination of variables, the system of equations (16)-(23) is reduced to the equations for $U_{R}, U_{\phi}, U_{z}$ :

$$
\begin{gathered}
\left(\gamma+\omega_{v}+\frac{\left(\vec{k} \vec{B}_{0}\right)^{2}}{4 \pi \rho_{0}\left(\gamma+\omega_{\eta}\right)}\right) U_{R}-2 \Omega \frac{k_{z}^{2}}{|\vec{k}|^{2}} U_{\phi}+g \beta \frac{A k_{z} k_{R} U_{z}}{|\vec{k}|^{2}\left(\gamma+\omega_{\chi}\right)}=0 \\
\left(2 \Omega(1+R o)+\frac{\left(\vec{k} \vec{B}_{0}\right)^{2}}{4 \pi \rho_{0}\left(\gamma+\omega_{\eta}\right)^{2}} R \frac{\partial \Omega}{\partial R}\right) U_{R}+\left(\gamma+\omega_{v}+\frac{\left(\vec{k} \vec{B}_{0}\right)^{2}}{4 \pi \rho_{0}\left(\gamma+\omega_{\eta}\right)}\right) U_{\phi}=0 \\
2 \Omega \frac{k_{z} k_{R}}{|\vec{k}|^{2}} U_{\phi}+\left(\gamma+\omega_{v}+\frac{\left(\vec{k} \vec{B}_{0}\right)^{2}}{4 \pi \rho_{0}\left(\gamma+\omega_{\eta}\right)}-\frac{g \beta A k_{R}^{2}}{|\vec{k}|^{2}\left(\gamma+\omega_{\chi}\right)}\right) U_{z}=0
\end{gathered}
$$

So, the task to provide the stability of a rotating magnetized flow with temperature stratification leads to the problem of finding the eigenvalues of $\gamma$ from the system of equations (24)-(26).

\section{Analysis of the dispersion equation}

The condition for solving the system of equations (24)-(26) is that its determinant equals zero, then we obtain the dispersion equation:

$$
\begin{gathered}
{\left[\left(\gamma+\omega_{v}\right)\left(\gamma+\omega_{\eta}\right)+\omega_{A}^{2}\right]^{2} \cdot\left[\left(\gamma+\omega_{v}\right)\left(\gamma+\omega_{\eta}\right)\left(\gamma+\omega_{\chi}\right)+\omega_{A}^{2}\left(\gamma+\omega_{\chi}\right)-N_{A}^{2}\left(1-\xi^{2}\right)\left(\gamma+\omega_{\eta}\right)\right]+} \\
+N_{A}^{2} \xi^{2}\left(1-\xi^{2}\right)\left(\gamma+\omega_{\eta}\right) \cdot\left[\kappa^{2}\left(\gamma+\omega_{\eta}\right)^{2}+\omega_{A}^{2}\left(\kappa^{2}-4 \Omega^{2}\right)\right]+\xi^{2} \cdot\left[\kappa^{2}\left(\gamma+\omega_{\eta}\right)^{2}+\omega_{A}^{2}\left(\kappa^{2}-4 \Omega^{2}\right)\right] \times \\
\times\left[\left(\gamma+\omega_{v}\right)\left(\gamma+\omega_{\eta}\right)\left(\gamma+\omega_{\chi}\right)+\omega_{A}^{2}\left(\gamma+\omega_{\chi}\right)-N_{A}^{2}\left(1-\xi^{2}\right)\left(\gamma+\omega_{\eta}\right)\right]=0
\end{gathered}
$$


Here $\omega_{A}$ is Alfven frequency, $\omega_{A}^{2}=k_{z}^{2} c_{A}^{2}=\frac{k_{z}^{2} B_{0}^{2}}{4 \pi \rho_{0}}, N_{A}=\sqrt{g \beta A}$ is Vaysel-Brent frequency, dependent on the temperature gradient, $\kappa=2 \Omega \sqrt{1+R o}$ is epicyclic frequency, $\xi=\frac{k_{z}}{|\vec{k}|}$.

Equation (27) after simple algebraic transformations splits into two dispersion equations of the following form:

$$
\begin{gathered}
\left(\gamma+\omega_{v}\right)\left(\gamma+\omega_{\eta}\right)+\omega_{A}^{2}=0 \\
\left(\gamma+\omega_{\chi}\right)\left[\left(\left(\gamma+\omega_{v}\right)\left(\gamma+\omega_{\eta}\right)+\omega_{A}^{2}\right)^{2}+\xi^{2} \kappa^{2}\left(\left(\gamma+\omega_{\eta}\right)^{2}+\omega_{A}^{2}\right)-4 \Omega^{2} \xi^{2} \omega_{A}^{2}\right]- \\
-N_{A}^{2}\left(1-\xi^{2}\right)\left(\gamma+\omega_{\eta}\right)\left[\left(\gamma+\omega_{v}\right)\left(\gamma+\omega_{\eta}\right)+\omega_{A}^{2}\right]=0
\end{gathered}
$$

The dispersion equation (28) describes the attenuation of Alfven waves in plasma with viscous and ohmic dissipation. In this equation, the influence of rotation and temperature stratification on the perturbation increment is not observed, therefore, we begin to analyze the dispersion equation (29). In some extreme cases, this equation gives the results, which are known by this time.

1). Let us consider the purely hydrodynamic limit, when the medium is homogeneous by temperature $(A=0)$, non-dissipative, and rotates at the angular velocity $\Omega=\Omega(R)$ (Couette flow) in the absence of the magnetic field, then from equation (29) we get:

$$
\gamma^{2}+\xi^{2} \kappa^{2}=0
$$

From the above it follows that the necessary and sufficient condition for the stability of the rotating shear flow (Rayleigh criterion, see, for example, the review in [39]) is the reality of the epicyclic frequency $\kappa^{2}>0$ or the realization of the inequality $R o>-1$. For the flow with the Rossby profile $R o=-1$, the axisymmetric perturbations in this extreme case are neutrally stable $\gamma=0$.

2). Taking into account the stratification by temperature $(A \neq 0)$ and $v=\chi=\eta=0$, from equation (29) we get:

$$
\gamma^{2}+\xi^{2} \kappa^{2}-N_{A}^{2} \frac{k_{R}^{2}}{|\vec{k}|^{2}}=0
$$

In this case, the temperature stratification can either stabilize $\left(N_{A}^{2}<0\right)$ or destabilize $\left(N_{A}^{2}>0\right)$ the stable Couette flow $\left(\kappa^{2}>0\right)$, depending on the direction of the temperature gradient.

3). Within the limits of the ideal magnetic hydrodynamics $(v=\chi=\eta=0)$ with $A=0, \vec{B}_{0} \neq 0, \vec{\Omega} \neq 0$, Chandrasekar [23] and Velikhov [24] have shown that the magnetic field destabilizes the Couette flow. Indeed, from equation (29) for this case we have:

$$
\left(\gamma^{2}+\omega_{A}^{2}\right)^{2}+\xi^{2} \kappa^{2}\left(\gamma^{2}+\omega_{A}^{2}\right)-4 \Omega^{2} \xi^{2} \omega_{A}^{2}=0
$$

or

$$
\gamma^{2}+\omega_{A}^{2}+\frac{\xi^{2} \kappa^{2}}{2}= \pm \sqrt{\frac{\xi^{4} \kappa^{4}}{4}+4 \Omega^{2} \xi^{2} \omega_{A}^{2}}
$$

From the above it follows that at moderate amplitudes of the magnetic field $\omega_{A}<\Omega$, the cumulative effect determined by the magnetic field is destabilizing, i.e. the development of instability currents at $\kappa^{2}>0$ is assumed to be possible. This effect is the cause of the standard MRI.

4). With the temperature stratification taken into account, the equation (32) will take the form:

$$
\left(\gamma^{2}+\omega_{A}^{2}\right)^{2}+\left(\xi^{2} \kappa^{2}-N_{A}^{2}\left(1-\xi^{2}\right)\right)\left(\gamma^{2}+\omega_{A}^{2}\right)-4 \Omega^{2} \xi^{2} \omega_{A}^{2}=0
$$

For the radially temperature-stratified plasma, when replacing $-N_{A}^{2}\left(1-\xi^{2}\right)$ by $\xi^{2} N^{2}$ $\left(N^{2}=-\frac{1}{\Gamma \rho_{0}} \frac{d p_{0}}{d R} \frac{d}{d R}\left(\ln \frac{p_{0}}{\rho_{0}^{\Gamma}}\right), \Gamma\right.$ is the adiabatic exponent), this equation was derived in [25] and actively studied in [40]. 
5). With only the rotation and the magnetic field in a homogeneous ( $A=0$ ) dissipative medium taken into account, the equation (29) is transformed into the dispersion equation of the following form:

$$
\left(\left(\gamma+\omega_{v}\right)\left(\gamma+\omega_{\eta}\right)+\omega_{A}^{2}\right)^{2}+\xi^{2} \kappa^{2}\left(\left(\gamma+\omega_{\eta}\right)^{2}+\omega_{A}^{2}\right)-4 \Omega^{2} \xi^{2} \omega_{A}^{2}=0
$$

This equation was studied in detail in [41-43], and its generalization, with the radial thermal stratification of the medium taken into account, was presented in [27].

6). In the absence of the rotation and the magnetic field, in the case of a nonconductive medium, from equation (29) we obtain the dispersion equation:

$$
\gamma^{2}+\gamma\left(\omega_{v}+\omega_{\chi}\right)+\omega_{\nu} \omega_{\chi}-N_{A}^{2}\left(1-\xi^{2}\right)=0
$$

When utilizing the dimensionless variables $\gamma \rightarrow \frac{v}{h^{2}} \gamma, k_{R} h \rightarrow k, k_{z} h \rightarrow \pi n$ in equation (36), we obtain the Rayleigh equation describing free convection in the liquid layer with thickness $h$. Its solution has the form, given in $[2]$ :

$$
\gamma_{n}=-\frac{(1+\operatorname{Pr})}{2 \operatorname{Pr}}\left(k^{2}+\pi^{2} n^{2}\right) \pm \sqrt{\left(\frac{(\operatorname{Pr}-1)}{2 \operatorname{Pr}}\right)^{2}\left(k^{2}+\pi^{2} n^{2}\right)^{2}+\frac{R a k^{2}}{\operatorname{Pr}\left(k^{2}+\pi^{2} n^{2}\right)}}
$$

where $n$ is an integral number characterizing the scale vertically. The magnitude of the instability increment $\gamma_{n}$ depends on the dimensionless numbers of Rayleigh $\operatorname{Ra}=\frac{g \beta A L^{4}}{v \chi}$, Prandtl $\operatorname{Pr}=\frac{v}{\chi}$, and the wave number $K=\sqrt{k^{2}+\pi^{2} n^{2}}$. The condition for the stability of small perturbations lies in the positiveness of the radicand, which corresponds to the Rayleigh numbers $R a>0$.

In contrast to [27], the dispersion equation (29), which we derived, takes into account the thermal dissipation (terms with $\omega_{\chi}$ ) and vertical stratification (terms with $N_{A}$ ) by temperature in the field of gravitation. Let us analyze the stability by writing down the dispersion equation (29) in the form of the fifth degree polynomial relative to $\gamma$ :

$$
P(\gamma) \equiv a_{0} \gamma^{5}+a_{1} \gamma^{4}+a_{2} \gamma^{3}+a_{3} \gamma^{2}+a_{4} \gamma+a_{5}=0,
$$

where the coefficients $a_{0}, a_{1}, a_{2}, a_{3}, a_{4}, a_{5}$ have the appropriate form:

$$
\begin{gathered}
a_{0}=1, \\
a_{1}=2\left(\omega_{\eta}+\omega_{v}\right)+\omega_{\chi}, \\
a_{2}=\left(\omega_{v}+\omega_{\eta}\right)^{2}+2\left(\omega_{A}^{2}+\omega_{\eta} \omega_{v}\right)+4 \xi^{2} \Omega^{2}(1+R o)-N_{A}^{2}\left(1-\xi^{2}\right)+2 \omega_{\chi}\left(\omega_{\eta}+\omega_{v}\right), \\
a_{3}=2\left(\omega_{v}+\omega_{\eta}\right)\left(\omega_{A}^{2}+\omega_{\eta} \omega_{v}\right)+8 \xi^{2} \Omega^{2}(1+R o)\left(\omega_{\eta}+\omega_{\chi} / 2\right)+\omega_{\chi}\left(\omega_{v}+\omega_{\eta}\right)^{2}+2 \omega_{\chi}\left(\omega_{A}^{2}+\omega_{v} \omega_{\eta}\right)- \\
-N_{A}^{2}\left(1-\xi^{2}\right)\left(2 \omega_{\eta}+\omega_{v}\right), \\
a_{4}=\left(\omega_{A}^{2}+\omega_{\eta} \omega_{v}\right)^{2}-4 \xi^{2} \Omega^{2} \omega_{A}^{2}+4 \xi^{2} \Omega^{2}(1+R o)\left(\omega_{\eta}^{2}+\omega_{A}^{2}\right)+8 \xi^{2} \Omega^{2}(1+R o) \omega_{\eta} \omega_{\chi}+2 \omega_{v} \omega_{\eta}^{2} \omega_{\chi}- \\
-N_{A}^{2}\left(1-\xi^{2}\right)\left(\omega_{\eta}^{2}+2 \omega_{v} \omega_{\eta}+\omega_{A}^{2}\right), \\
a_{5}=\omega_{\chi}\left(\left(\omega_{A}^{2}+\omega_{\eta} \omega_{v}\right)^{2}+4 \xi^{2} \Omega^{2} \omega_{\eta}^{2}+4 \xi^{2} \Omega^{2} R o\left(\omega_{\eta}^{2}+\omega_{A}^{2}\right)\right)-N_{A}^{2}\left(1-\xi^{2}\right)\left(\omega_{\eta} \omega_{A}^{2}+\omega_{\eta}^{2} \omega_{v}\right) .
\end{gathered}
$$

The dispersion equation (39) is the equation of the fifth degree relative to $\gamma$, so the analytical determination of its roots in general case is not possible. However, the conclusion about stability of the perturbations described by equation (39) with real coefficients can be made without solving it, but only by analyzing its coefficients using the RouthHurwitz or Lienar-Shipar criteria [44]. In the latter criterum the number of determinant inequalities is approximately half as much as in that of Routh-Hurwitz, therefore its application is advisable. The Liénard-Chipart criterion for the perturbations asymptotic stability, described by the algebraic equation (38), is as follows. For $P(\gamma)$ polynomial to have all roots with negative real parts, it is necessary and sufficient that: 
a) all the coefficients of $P(\gamma)$ polynomial were positive: $a_{n}>0, n=0 \ldots 5$;

b) the inequalities for the Hurwitz determinants were satisfied: $\Delta_{n-1}>0, \Delta_{n-3}>0 \ldots$, where $\Delta_{m}$ denoted the Hurwitz determinant of $m$ order:

$$
\Delta_{m}=\left|\begin{array}{ccccc}
a_{1} & a_{3} & a_{5} & \cdot & . \\
a_{0} & a_{2} & a_{4} & \cdot & . \\
0 & a_{1} & a_{3} & \cdot & . \\
0 & a_{0} & a_{2} & \cdot & . \\
\cdot & . & \cdot & \cdot & a_{m}
\end{array}\right|
$$

By using the algorithm of Leenard-Shepard we obtain necessary and sufficient conditions for stability of the nonuniformly rotating plasma with the constant temperature gradient:

$$
a_{n}>0, \quad n=0 \ldots 5, \quad \Delta_{2}>0, \quad \Delta_{4}>0
$$

Here, the determinants $\Delta_{2}$ and $\Delta_{4}$ are correspondingly equal:

$$
\begin{aligned}
\Delta_{2} & =\left|\begin{array}{ll}
a_{1} & a_{3} \\
1 & a_{2}
\end{array}\right|=a_{1} a_{2}-a_{3}, \\
\Delta_{4} & =\left|\begin{array}{llll}
a_{1} & a_{3} & a_{5} & 0 \\
1 & a_{2} & a_{4} & 0 \\
0 & a_{1} & a_{3} & a_{5} \\
0 & 1 & a_{2} & a_{4}
\end{array}\right|=a_{4}\left\{a_{3}\left(a_{1} a_{2}-a_{3}\right)-a_{1}^{2} a_{4}+2 a_{1} a_{5}\right\}-a_{5}\left\{a_{2}\left(a_{1} a_{2}-a_{3}\right)+a_{5}\right\}
\end{aligned}
$$

Substituting the values of coefficients $a_{n}$ from (39), into the conditions of (40) we find the following inequalities:

1) $\left(a_{1}>0\right) \Rightarrow 2\left(\omega_{\eta}+\omega_{v}\right)+\omega_{\chi}>0$, this inequality is performed automatically;

2) $\left(a_{2}>0\right) \Rightarrow\left(\omega_{v}+\omega_{\eta}\right)^{2}+2\left(\omega_{A}^{2}+\omega_{\eta} \omega_{v}\right)+4 \xi^{2} \Omega^{2}(1+R o)+2 \omega_{\chi}\left(\omega_{\eta}+\omega_{v}\right)>N_{A}^{2} \frac{k_{R}^{2}}{|\vec{k}|^{2}}$

This inequality shows that viscous, ohmic and thermal conductivity dissipation naturally lead to stabilization of the plasma flows stability. The stabilizing factors are also: the uniform magnetic field (Alfven effect), non-uniform rotation (if the profile of the angular velocity of rotation is close to $\Omega(R) \sim R^{-2}\left(\kappa^{2}>0\right)$ and the temperature gradient at $A<0$. In the limits of the nondissipative and homogeneous electrically conducting fluid in the uniform magnetic field this inequality transforms into the well-known Velikhov stability criterion [24]:

$$
\omega_{A}^{2}+\frac{\xi^{2} \kappa^{2}}{2}>0
$$

3) inequality $a_{3}>0$ :

$$
\begin{gathered}
2\left(\omega_{v}+\omega_{\eta}\right)\left(\omega_{A}^{2}+\omega_{\eta} \omega_{v}\right)+8 \xi^{2} \Omega^{2}(1+R o)\left(\omega_{\eta}+\omega_{\chi} / 2\right)+ \\
+\omega_{\chi}\left(\omega_{v}+\omega_{\eta}\right)^{2}+2 \omega_{\chi}\left(\omega_{A}^{2}+\omega_{v} \omega_{\eta}\right)>N_{A}^{2} \frac{k_{R}^{2}}{|\vec{k}|^{2}}\left(2 \omega_{\eta}+\omega_{v}\right)
\end{gathered}
$$

does not contain any new conditions for stabilization of disturbances;

4) inequality $a_{4}>0$ :

$$
\begin{gathered}
\left(\omega_{A}^{2}+\omega_{\nu} \omega_{\eta}\right)^{2}+2 \omega_{\nu} \omega_{\eta}^{2} \omega_{\chi}+4 \xi^{2} \Omega^{2} R o \omega_{A}^{2}+ \\
+4 \xi^{2} \Omega^{2}(1+R o)\left(\omega_{\eta}^{2}+2 \omega_{\nu} \omega_{\chi}\right)>N_{A}^{2} \frac{k_{R}^{2}}{|k|^{2}}\left(\omega_{\eta}^{2}+2 \omega_{\nu} \omega_{\eta}+\omega_{A}^{2}\right)
\end{gathered}
$$

5) $a_{5}>0$, or 


$$
1-\frac{R o}{R o_{c r}}+\frac{R o_{T}}{R o_{c r}} \cdot \frac{k_{R}^{2}}{k_{z}^{2}} \cdot \frac{\omega_{A}^{2} \omega_{\eta}+\omega_{\nu} \omega_{\eta}^{2}}{\left(\omega_{A}^{2}+\omega_{\eta}^{2}\right) \omega_{\chi}}>0, R o_{c r}=-\frac{\left(\omega_{A}^{2}+\omega_{\nu} \omega_{\eta}\right)^{2}+4 \xi^{2} \Omega^{2} \omega_{\eta}^{2}}{4 \Omega^{2} \xi^{2}\left(\omega_{A}^{2}+\omega_{\eta}^{2}\right)}
$$

The parameter $R o_{c r}$ in inequality (42) corresponds to the critical value of the Rossby number $R o$ for the standard MRI (SMRI), which was obtained in [29]. The dimensionless parameters: $R o_{T}=N_{A}^{2} / 4 \Omega^{2}=R a / T a P r$ is the thermal number of Rossby, the numbers: $R a=g \beta A L^{4} / v \chi$ - of Rayleigh, $T a=4 \Omega^{2} L^{4} / v^{2}$ - of Taylor on the typical scale of stratification $L, \operatorname{Pr}=v / \chi$ is the Prandtl number.

Now we turn to the stability conditions b) consisting of inequalities with Hurwitz determinants (40). For the determinant $\Delta_{2}>0$ we get:

$$
\frac{2\left(\omega_{\eta}^{3}+\omega_{v}^{3}\right)+8 \omega_{\nu} \omega_{\eta}\left(\omega_{\eta}+\omega_{\nu}+\omega_{\chi}\right)+3 \omega_{\chi}\left(\omega_{\eta}^{2}+\omega_{v}^{2}\right)+2 \omega_{A}^{2}\left(\omega_{v}+\omega_{\eta}\right)}{\omega_{v}+\omega_{\chi}}-\frac{2 \omega_{A}^{2} \omega_{\chi}}{\omega_{v}+\omega_{\chi}}>g \beta A \frac{k_{R}^{2}}{k^{2}}
$$

In this inequality a new destabilizing term (the second fraction) has appeared, which has a significant impact, provided that the Prandtl number $\operatorname{Pr} \ll 1$ is small. Under the condition $\operatorname{Pr} \approx 1$ and $\operatorname{Pr} \gg 1$ the perturbations stabilization by the magnetic field in a dissipative medium takes place. After substituting the values of coefficients $a_{n}$ into the expression for the Hurwitz determinant $\Delta_{4}$, we obtain the last of the stability conditions: $\Delta_{4}>0$. We do not give the explicit form of inequality $\Delta_{4}>0$ because of the cumbersome form of the included expressions. However, note that the stability criterion $\Delta_{4}>0$ contains the previous stability criterion (43).

\section{RAYLEIGH-BENARD PROBLEM FOR A THIN LAYER OF THE INHOMOGENEOUSLY ROTATING MAGNETIC PLASMA \\ Formulation of the problem and basic equations}

The system of equations (6) obtained in the previous section will be used to describe convective phenomena in the thin layer of the inhomogeneously rotating conducting medium (plasma) with thickness $h \ll\left(R_{\text {out }}-R_{\text {in }}\right)$. The temperature of the lower part of the layer is denoted by $T_{d}$, and the upper one - by $T_{u}$, while $T_{d}>T_{u}$ is the heating from the bottom (Fig. 3).

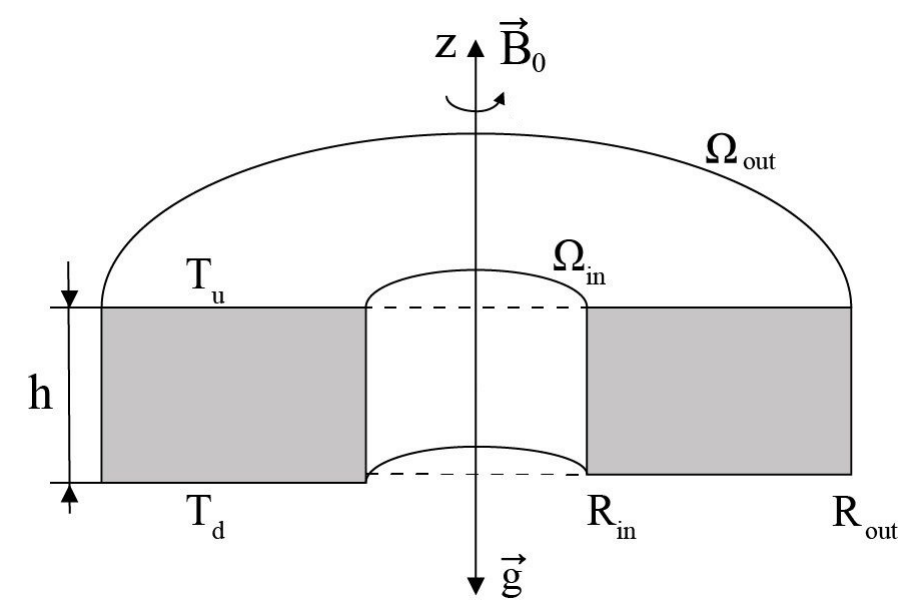

Fig. 3. The geometry of the problem for the inhomogeneously rotating magnetoconvection. The electrically conductive fluid fills the layer between two rotating cylinders with angular velocities $\Omega_{\text {in }}$ and $\Omega_{\text {out }}$, respectively. The bottom surface of the layer has the temperature $T_{d}$, and the top one $-T_{u}: T_{d}>T_{u}$.

Such a formulation of the problem generalizes the classical Rayleigh-Benard problem for free convection. For this problem the typical scale of the medium inhomogeneity in the horizontal plane is larger than in the vertical direction $L_{R} \gg L_{h}$. Therefore, we will be able to apply the local WKB method for the disturbances depending on the horizontal coordinates $(R, \phi)$. We expand all the quantities into the Taylor series in the vicinity of the fixed points $\left(R_{0}, \phi_{0}\right)$, leaving the terms of the zero order in the local coordinates $\tilde{R}=R-R_{0}, \tilde{\varphi}=\varphi-\varphi_{0}$. As a result, we obtain the system of differential equations (6) with constant coefficients. In this case the following relations will be taken into account: 


$$
\begin{gathered}
\Omega_{0}=\Omega\left(R_{0}\right), \nabla^{2} \rightarrow \bar{D}^{2}+\frac{\partial^{2}}{\partial \tilde{R}^{2}}+\frac{1}{R_{0}} \frac{\partial}{\partial \tilde{R}}+\frac{1}{R_{0}^{2}} \frac{\partial}{\partial \tilde{\varphi}^{2}}, \hat{D} \equiv \frac{d}{d z}, \\
\left(\nabla^{2}\left(\begin{array}{l}
u \\
b
\end{array}\right)\right)_{R}=\nabla^{2}\left(\begin{array}{l}
u_{R} \\
b_{R}
\end{array}\right)-\frac{2}{R_{0}^{2}} \frac{\partial}{\partial \phi}\left(\begin{array}{l}
u_{\phi} \\
b_{\phi}
\end{array}\right)-\frac{1}{R_{0}^{2}}\left(\begin{array}{l}
u_{R} \\
b_{R}
\end{array}\right), \\
\left(\nabla^{2}\left(\begin{array}{l}
u \\
b
\end{array}\right)\right)_{\phi}=\nabla^{2}\left(\begin{array}{l}
u_{\phi} \\
b_{\phi}
\end{array}\right)+\frac{2}{R_{0}^{2}} \frac{\partial}{\partial \phi}\left(\begin{array}{l}
u_{R} \\
b_{R}
\end{array}\right)-\frac{1}{R_{0}^{2}}\left(\begin{array}{l}
u_{\phi} \\
b_{\phi}
\end{array}\right) .
\end{gathered}
$$

Like in the previous section, our studies will be restricted to the axisymmetric perturbations $\partial / \partial \phi=0$. Then all the perturbations in the system of equations (6) can be represented in the form of plane waves.

$$
\left(\begin{array}{c}
\vec{u} \\
\vec{b} \\
\theta \\
\tilde{p}
\end{array}\right)=\left(\begin{array}{c}
\vec{U}(z) \\
\vec{H}(z) \\
\Theta(z) \\
\tilde{P}(z)
\end{array}\right) \exp (\gamma t+i k \tilde{R})
$$

then, in the short-wave approximation $k \gg \frac{1}{R_{0}}$, neglecting the terms $\frac{i k}{R_{0}}-\frac{1}{R_{0}^{2}}$, we obtain

$$
\begin{aligned}
& \left(\widehat{D}^{2}-\gamma-k^{2}\right) U_{R}+\sqrt{T a} U_{\varphi}+\operatorname{Pr} P m^{-1} H a^{2} \widehat{D} H_{R}-i k \tilde{P}=0 \\
& \left(\widehat{D}^{2}-\gamma-k^{2}\right) U_{\varphi}-\sqrt{T a}(1+R o) U_{R}+\operatorname{Pr} P m^{-1} H a^{2} \widehat{D} H_{\varphi}=0 \\
& \left(\widehat{D}^{2}-\gamma-k^{2}\right) U_{z}+\operatorname{Pr} P m^{-1} H a^{2} \widehat{D} H_{z}+R a \Theta-\hat{D} \tilde{P}=0 \\
& \left(\widehat{D}^{2}-P m \gamma-k^{2}\right) H_{R}+\operatorname{Pr}^{-1} \operatorname{Pm} \hat{D} U_{R}=0 \\
& \left(\widehat{D}^{2}-P m \gamma-k^{2}\right) H_{\varphi}+\operatorname{Pr}^{-1} P m \hat{D} U_{\varphi}+\sqrt{T a} P m R o H_{R}=0 \\
& \left(\widehat{D}^{2}-P m \gamma-k^{2}\right) H_{z}+\operatorname{Pr}^{-1} \operatorname{Pm} \hat{D} U_{z}=0 \\
& \left(\widehat{D}^{2}-\operatorname{Pr} \gamma-k^{2}\right) \Theta+U_{z}=0, \\
& \hat{D} U_{z}+i k U_{R}=0, \hat{D} H_{z}+i k H_{R}=0 \text {, }
\end{aligned}
$$

The system of equations (45)-(52) is written down in a dimensionless form, in which the dimensionless values retain the form of dimensional ones:

$$
\begin{gathered}
z \rightarrow h^{-1} z,\left(U_{R}, U_{\phi}, U_{z}\right) \rightarrow \chi h^{-1}\left(U_{R}, U_{\phi}, U_{z}\right),\left(H_{R}, H_{\phi}, H_{z}\right) \rightarrow B_{0}^{-1}\left(H_{R}, H_{\phi}, H_{z}\right), \\
\Theta \rightarrow \Theta(A h)^{-1}, \tilde{P} \rightarrow \tilde{P}\left(\frac{h^{2}}{\rho_{0} v \chi}\right), t \rightarrow t\left(\frac{v}{h^{2}}\right), \frac{\partial}{\partial t} \rightarrow \frac{h^{2}}{v} \frac{\partial}{\partial t} .
\end{gathered}
$$

In equations (45)-(52) the following dimensionless parameters are introduced $\operatorname{Pr}=v / \chi$ is the Prandtl number, $P m=v / \eta$ is the Prandtl magnetic number, the numbers: $T a=4 \Omega_{0}{ }^{2} h^{4} / v^{2}$ is of Taylor, $H a=B_{0} h / \sqrt{4 \pi \rho_{0} v \eta}$ is of Hartmann, $R a=g \beta A h^{4} / v \chi$ is of Rayleigh on the scale $h$. Then, instead of the Hartman number $H a$ we will use the Chandrasekhar number $Q=H a^{2}$. Using the equation of the solenoidal character of fields (52), we exclude pressure $\tilde{P}$ from equations (45) and (46):

$$
\tilde{P}=R a \cdot \frac{\widehat{D} \Theta}{\widehat{D}^{2}-k^{2}}+\frac{i k \sqrt{T a}}{\widehat{D}^{2}-k^{2}} \cdot U_{\varphi}
$$


In order to reduce the number of variables, we introduce indications for $z$-component of the vortex $\xi=(\operatorname{rot} U)_{z}=i k U_{\phi}$, of the current $-\zeta=(\operatorname{rot} H)_{z}=i k H_{\phi}$ and for $z$-component of the velocity $-W=U_{z}$. With the new indications taken into account equations (45)-(52) will take the form:

$$
\begin{gathered}
\left(\widehat{D}^{2}-k^{2}\right)\left(\widehat{D}^{2}-k^{2}-\gamma\right) W+\tilde{Q}\left(\widehat{D}^{2}-k^{2}\right) \hat{D} H_{z}-\sqrt{T a} \hat{D} \zeta=k^{2} R a \Theta \\
\left(\widehat{D}^{2}-k^{2}-\gamma\right) \zeta+\sqrt{T a}(1+R o) \hat{D} W+\tilde{Q} \hat{D} \xi=0 \\
\left(\widehat{D}^{2}-P m \gamma-k^{2}\right) H_{z}+\operatorname{Pr}^{-1} \operatorname{Pm} \hat{D} W=0 \\
\left(\widehat{D}^{2}-P m \gamma-k^{2}\right) \xi+\operatorname{Pr}^{-1} \operatorname{Pm} \hat{D} \zeta-\sqrt{T a} \operatorname{PmRoD} H_{z}=0 \\
\left(\widehat{D}^{2}-\operatorname{Pr} \gamma-k^{2}\right) \Theta+W=0,
\end{gathered}
$$

where $\tilde{Q}=\operatorname{Pr} P m^{-1} Q$. We complete equations (54)-(58) with the following boundary conditions: for "free" (free-free boundaries) surfaces at $z=0,1$ :

$$
W=\Theta=\widehat{D} H_{z}=0, \widehat{D}^{2} W=\widehat{D} \zeta=\xi=0
$$

and for "rigid" (rigid-rigid boundaries) surfaces at $z=0,1$ :

$$
W=\Theta=H_{z}=0, \hat{D} W=\zeta=\hat{D} \xi=0
$$

Equations (54)-(58) with boundary conditions of (59)-(60) describe the linear (for small perturbations) convection in the thin layer of the non-uniformly rotating magnetized fluid.

\section{STATIONARY CONVECTION FOR FREE BOUNDARIES Chandrasekhar variation principle}

Let us consider the stationary convection mode, i.e. when the system is in a neutral state $\gamma=0$. In this case, the problem of the eigenvalues of equations (54)-(58) lies in finding the critical Rayleigh numbers $R a$ that satisfy the following equation:

$$
\begin{gathered}
{\left[\left(\widehat{D}^{2}-k^{2}\right)\left(\left(\widehat{D}^{2}-k^{2}\right)^{2}-Q \widehat{D}^{2}\right)^{2}+T a(1+R o)\left(\widehat{D}^{2}-k^{2}\right)^{2} \widehat{D}^{2}-\operatorname{TaRoPm} Q \widehat{D}^{4}\right] W=} \\
=-k^{2} \operatorname{Ra}\left(\left(\widehat{D}^{2}-k^{2}\right)^{2}-Q \widehat{D}^{2}\right) W
\end{gathered}
$$

with boundary conditions: $W=\widehat{D}^{(p)} W=0(p=2,4,6,8,10)$ at $z=0.1$. According to these boundary conditions, the even derivatives of the function $W$ at the surface boundaries $z=0$ and $z=1$ should become zero.

We formulate the problem of finding the eigenvalues (the Rayleigh number $R a$ ) of equation (61) using the Chandrasekhar variational principle. For this, from the system of equations (54)-(58), provided that $\gamma=0$, we exclude the perturbations for the components of the vortex $\xi$ and current $\zeta$. As a result we get:

$$
\begin{gathered}
\widehat{L}\left(\widehat{D}^{2}-k^{2}\right)^{2} W+\tilde{Q} \hat{L}\left(\widehat{D}^{2}-k^{2}\right) \hat{D} H_{z}-\sqrt{T a} \widehat{D} \hat{L} \zeta=k^{2} R a \hat{L} \Theta \\
\widehat{L} \zeta=-\sqrt{T a}(1+R o)\left(\widehat{D}^{2}-k^{2}\right) \hat{D} W-\sqrt{T a} Q \operatorname{Pr} R o \widehat{D}^{2} H_{z}, \\
\left(\widehat{D}^{2}-k^{2}\right) H_{z}+\operatorname{Pr}^{-1} \operatorname{Pm} \hat{D} W=0 \\
\left(\widehat{D}^{2}-k^{2}\right) \Theta+W=0,
\end{gathered}
$$

where the operator is $\widehat{L}=\left(\widehat{D}^{2}-k^{2}\right)^{2}-Q \widehat{D}^{2}$.

We multiply the equation (62) from the left by $W$ and integrate it by $z$ :

$$
\int_{0}^{1} W \widehat{L}\left(\widehat{D}^{2}-k^{2}\right)^{2} W d z+\tilde{Q} \int_{0}^{1} W \widehat{L}\left(\widehat{D}^{2}-k^{2}\right) \hat{D} H_{z} d z+T a(1+R o) \int_{0}^{1} W\left(\widehat{D}^{2}-k^{2}\right) \widehat{D}^{2} W d z+
$$




$$
+\operatorname{TaQPrRo} \int_{0}^{1} W \widehat{D}^{3} H_{z} d z=k^{2} \operatorname{Ra} \int_{0}^{1} W \widehat{L} \Theta d z
$$

With equations (64) and (65) taken into account, equation (66) takes the form:

$$
\begin{aligned}
& \int_{0}^{1} W\left(\widehat{L}^{2}+T a(1+R o)\left(\widehat{D}^{2}-k^{2}\right) \widehat{D}^{2}\right) W d z+\frac{\operatorname{Pr}^{2}}{P m} \operatorname{TaQRo} \int_{0}^{1}\left[\left(\widehat{D}^{2} H_{z}\right)^{2}-k^{2} H_{z} \widehat{D}^{2} H_{z}\right] d z= \\
& =-k^{2} \operatorname{Ra} \int_{0}^{1}\left(\widehat{D}^{2}-k^{2}\right) \Theta \widehat{L} \Theta d z
\end{aligned}
$$

In equation (67) we carry out partial integration for several times using the boundary conditions of (59). As a result, we obtain` the expression for $R a$, as the ratio of positive definite integrals:

$$
R a=\frac{1}{k^{2} I_{4}}\left(I_{1}+T a(1+R o) \cdot I_{2}+\frac{\operatorname{Pr}^{2}}{P m} T a Q R o \cdot I_{3}\right),
$$

where indications for integrals $I_{1,2,3,4}>0$ are introduced:

$$
\begin{gathered}
I_{1}=\int_{0}^{1}\left[\left(\widehat{D}^{4} W\right)^{2}+6 k^{4}\left(\widehat{D}^{2} W\right)^{2}+4 k^{2}\left(\widehat{D}^{3} W\right)^{2}+4 k^{6}(\widehat{D} W)^{2}+k^{8} W^{2}\right] d z+ \\
+2 Q \int_{0}^{1}\left[\left(\widehat{D}^{3} W\right)^{2}+2 k^{2}\left(\widehat{D}^{2} W\right)^{2}+k^{4}(\widehat{D} W)^{2}\right] d z+Q^{2} \int_{0}^{1}\left(\widehat{D}^{2} W\right)^{2} d z \\
I_{2}=\int_{0}^{1}\left[\left(\widehat{D}^{2} W\right)^{2}+k^{2}(\widehat{D} W)^{2}\right] d z, \quad I_{3}=\int_{0}^{1}\left[\left(\widehat{D}^{2} H_{z}\right)^{2}+k^{2}\left(\widehat{D} H_{z}\right)^{2}\right] d z \\
I_{4}=\int_{0}^{1}\left[\left(\widehat{D}^{3} \Theta\right)^{2}+3 k^{2}\left(\widehat{D}^{2} \Theta\right)^{2}+3 k^{4}(\widehat{D} \Theta)^{2}+k^{6} \Theta^{2}+Q \int_{0}^{1}\left[\left(\widehat{D}^{2} \Theta\right)^{2}+k^{2}(\widehat{D} \Theta)^{2} d z .\right.\right.
\end{gathered}
$$

Now we will consider the variation of the Rayleigh number $\delta R a$ subject to the variations $\delta W, \delta H_{z}, \delta \Theta$. In the first order of the variations smallness we get:

$$
\begin{array}{r}
\delta R a=\frac{1}{k^{2} I_{4}}\left[\delta I_{1}+\operatorname{Ta}(1+R o) \cdot \delta I_{2}+\frac{\operatorname{Pr}^{2}}{P m} \operatorname{TaQRo} \cdot \delta I_{3}\right]- \\
\quad-\left(I_{1}+T a(1+R o) \cdot I_{2}+\frac{\operatorname{Pr}^{2}}{P m} T a Q R o \cdot I_{3}\right) \frac{\delta I_{4}}{k^{2} I_{4}^{2}}= \\
=\frac{1}{k^{2} I_{4}}\left[\delta I_{1}+T a(1+R o) \cdot \delta I_{2}+\frac{\operatorname{Pr}^{2}}{P m} \operatorname{TaQRo} \cdot \delta I_{3}-k^{2} R a \cdot \delta I_{4}\right]
\end{array}
$$

Next, we find variations of the integrals $\delta I_{1,2,3,4}$.

$$
\delta I_{1}=2 \int_{0}^{1} \widehat{L}^{2} W \cdot \delta W d z, \delta I_{2}=2 \int_{0}^{1} \widehat{D}^{2}\left(\widehat{D}^{2}-k^{2}\right) W \cdot \delta W d z
$$




$$
\delta I_{3}=2 \int_{0}^{1} \widehat{D}^{2}\left(\widehat{D}^{2}-k^{2}\right) H_{z} \cdot \delta H_{z} d z, \delta I_{4}=-2 \int_{0}^{1}\left(\widehat{D}^{2}-k^{2}\right) \widehat{L} \Theta \cdot \delta \Theta d z
$$

and then we substitute them into equation (69). Using equations (64)-(65) and boundary conditions of (59), we obtain the final expression for $\delta R a$ :

$$
\begin{gathered}
\delta R a=\frac{2}{k^{2} I_{4}} \times \\
\times\left\{\int_{0}^{1}\left[\left(\widehat{D}^{2}-k^{2}\right) \widehat{L}^{2} W+T a(1+R o)\left(\widehat{D}^{2}-k^{2}\right)^{2} \widehat{D}^{2} W-T a R o P m Q \widehat{D}^{4} W+k^{2} R a \hat{L} W\right] \cdot \frac{\delta W d z}{\left(\widehat{D}^{2}-k^{2}\right)}\right\}
\end{gathered}
$$

From the above it follows that $\delta R a=0$ for any arbitrary variation $\delta W \neq 0$, if

$$
\left(\widehat{D}^{2}-k^{2}\right) \widehat{L}^{2} W+\operatorname{Ta}(1+R o)\left(\widehat{D}^{2}-k^{2}\right)^{2} \widehat{D}^{2} W-\operatorname{TaRoPm} Q \widehat{D}^{4} W=-k^{2} R a \hat{L} W,
$$

i.e. equation (60) is satisfied. Then the function $W$, by which $R a$ is expressed (see equation (68)), is a solution for the problem of the characteristic values of equation (60).

\section{Exact solutions to the problem of characteristic values}

We choose a function $W$, that satisfies the free boundary conditions of (59), in the following form:

$$
W=W_{0} \sin n \pi z \quad(n=1,2,3 \ldots),
$$

where $W_{0}=$ const is the disturbances amplitude of $z$ - velocity component.

By restricting to the single-mode approximation $(n=1)$, while substituting (71) into (76), we obtain the expression for the critical value of the Rayleigh number $R a_{c}$ of stationary convection:

$$
R a_{c}=\frac{\left(\pi^{2}+k^{2}\right)^{3}}{k^{2}}+\frac{\pi^{2}\left(\pi^{2}+k^{2}\right) Q}{k^{2}}+\frac{\pi^{2}\left(\pi^{2}+k^{2}\right)^{2} T a}{k^{2}\left(\left(\pi^{2}+k^{2}\right)^{2}+\pi^{2} Q\right)}+\frac{\pi^{2} T a R o\left(\left(\pi^{2}+k^{2}\right)^{2}+\pi^{2} Q P m\right)}{k^{2}\left(\left(\pi^{2}+k^{2}\right)^{2}+\pi^{2} Q\right)}
$$

In the new variables

$$
x=\frac{k^{2}}{\pi^{2}}, \quad Q_{1}=\frac{Q}{\pi^{2}}, \quad T_{1}=\frac{T a}{\pi^{4}}, \quad R_{1}=\frac{R a_{c}}{\pi^{4}},
$$

introduced by Chandrasekhar [1], equation (72) takes the form:

$$
R_{1}=\frac{(1+x)\left((1+x)^{2}+Q_{1}\right)^{2}+(1+x)^{2}(1+R o) T_{1}+\operatorname{RoPm} Q_{1} T_{1}}{x\left((1+x)^{2}+Q_{1}\right)}
$$

The function $R_{1}(x)$ takes extreme values for the corresponding $x$, which satisfy the following equation:

$$
2 x^{3}+3 x^{2}-1=Q_{1}+T_{1}(1+R o) \cdot \frac{(1+x)^{4}-\left(x^{2}-1\right) Q_{1}}{\left((1+x)^{2}+Q_{1}\right)^{2}}+\frac{R o P m Q_{1} T_{1}\left((1+x)(1+3 x)+Q_{1}\right)}{\left((1+x)^{2}+Q_{1}\right)^{2}}
$$

Figure 4 shows diagrams of the dependence of the stationary Rayleigh number $R_{1}$, determined by equation (73), on the wave numbers $x=k^{2} / \pi^{2}$ for the fixed parameters of the magnetic field $Q_{1}$ and of the rotation $T_{1}$. Curves 1,2,3 in Fig. 4 correspond to the case of the uniform (or solid-state) rotation $R o=0$, and they completely agree with the results of Chandrasekhar [1]. 


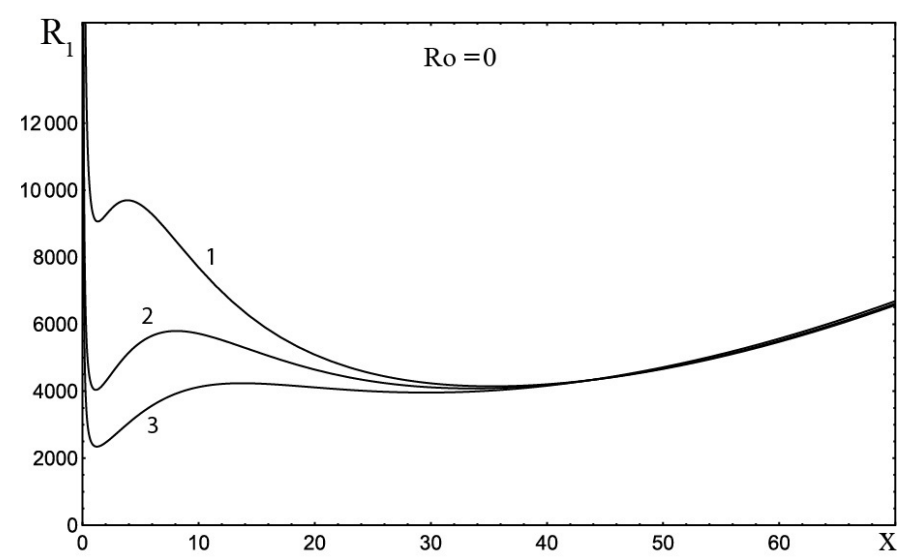

Fig. 4. Dependence of the Rayleigh number $R_{1}$ on the wave numbers $x=k^{2} / \pi^{2}$ for the Rossby numbers $R o=0$ with constant parameters: curve $1-Q_{1}=40, T_{1}=10^{5}$; curve $2-Q_{1}=100, T_{1}=10^{5}$; curve $3-Q_{1}=200, T_{1}=10^{5}$.

The diagrams in Figures 5 and 6 show the dependence of the Rayleigh number $R a_{c}$ on $\pi / k$, and are plotted for different Rossby numbers $R o$. The diagram in Fig. 5 corresponds to the parameters $T a=500, Q=100$. Here we can see that with an increase in the positive profile of the Rossby number $R o$, the minimum value of the critical Rayleigh number also increases, i.e. the threshold of the instability development rises. On the other hand, for the negative rotation profiles: of Keplerian $(R o=-3 / 4)$ and of Rayleigh $(R o=-1)$, we observe a decrease in the critical Rayleigh number, i.e. a lower threshold of the instability development, as compared to the case of the uniform $(R o=0)$ and non-uniform $(R o=2)$ rotation. The diagrams in Fig. 6a,b are plotted respectively for Taylor numbers $T a=10^{4}$ and $T a=10^{5}$. From these diagrams it follows that with the rotation increase (Taylor number $T a$ ) with a negative Rossby profile $(R o<0)$, no extreme values of the Rayleigh number are observed.

Now let us find out how the inhomogeneous rotation affects the process of stationary convection by calculating the derivative $d R_{1} / d R o$ :

$$
f(x)=\frac{d R_{1}}{d R o}=\frac{T_{1}\left((1+x)^{2}+Q_{1} P m\right)}{x\left((1+x)^{2}+Q_{1}\right)} .
$$

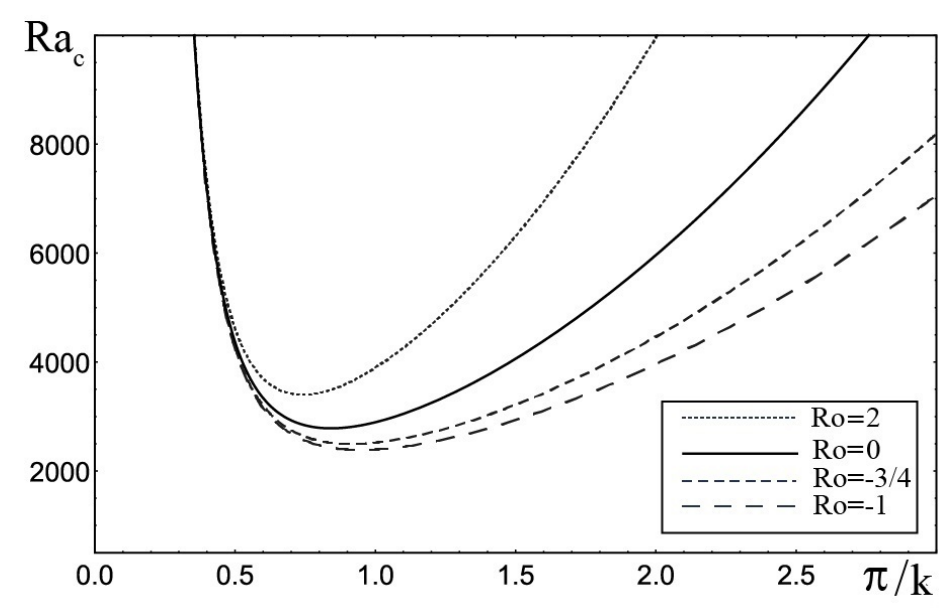

Fig. 5. Dependence of the Rayleigh number $R a_{c}$ on $\pi / k$ for different Rossby numbers $R o$ with constant parameters: $Q=100$, $T a=500$. The Prandtl magnetic number is assumed to be equal to one: $P m=1$.

The function graph $f(x)$ is shown in Fig. 7. It shows that with the increase in the Taylor number $T_{1}$ (from 5000 to $10^{5}$ ) at the fixed value of the magnetic field $Q_{1}=100$, the rate of variation of the value $d R_{1} / d R o$ increases towards small $x$ (long-wave disturbances $\sim k^{-1}$ ), which is true for positive values of the Rossby number $R o>0$. 


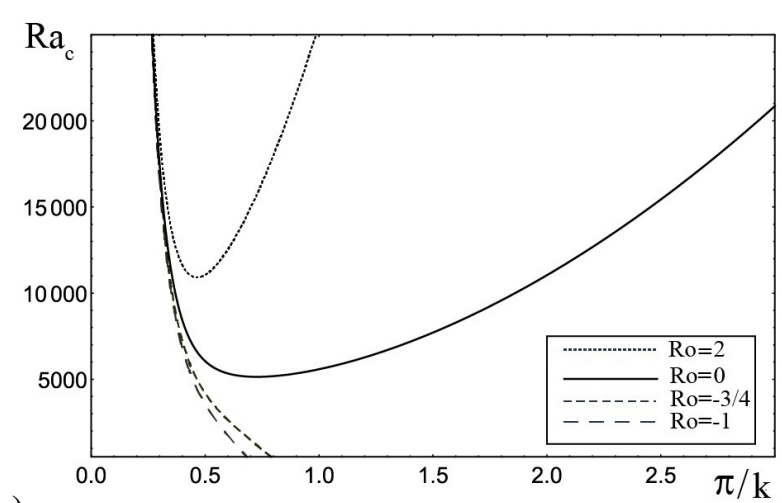

a)

Fig. 6. Dependence of Rayleigh number $R a_{c}$ on $\pi / k$ for different Rossby numbers $R o$ with constant parameters: a) $Q=100$, $T a=10^{4}$. b) $Q=100, T a=10^{5}$. The magnetic Prandtl number is assumed to be equal to one: $P m=1$.

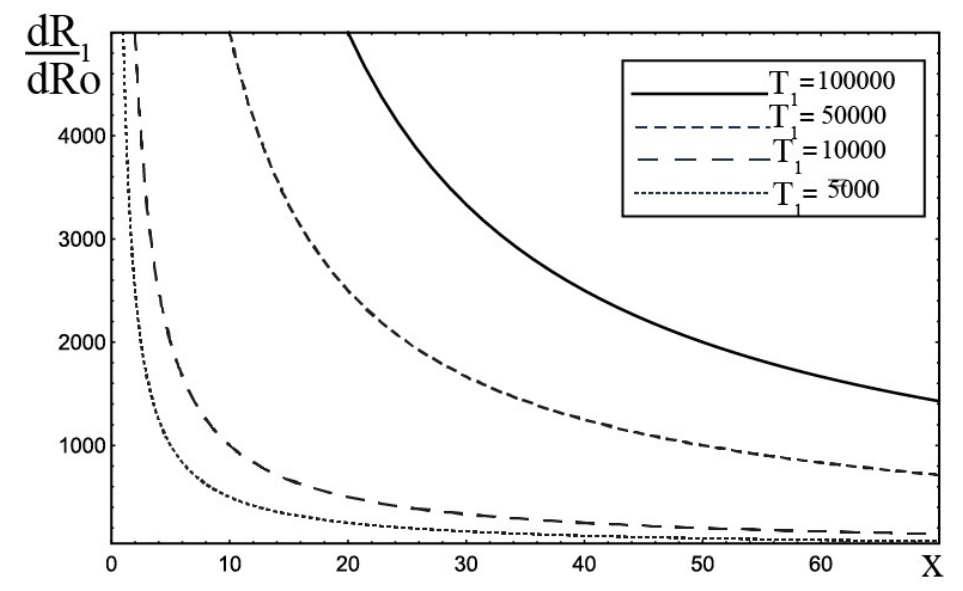

Fig. 7. The diagram of the $d R_{1} / d R o$ variation rate dependence on the wave numbers $x=k^{2} / \pi^{2}$ for different values of the Taylor number $T_{1}$ with constant parameters $Q_{1}=100$ and $P m=1$.

Fig. 8 shows graphs of variations in the Rayleigh number $R_{1}$ from the value $R o$ in the interval $[-1,2]$. These grahs show that the rotation with a negative angular velocity profile $R o<0$ has a destabilizing effect on the development of stationary convection.

a)

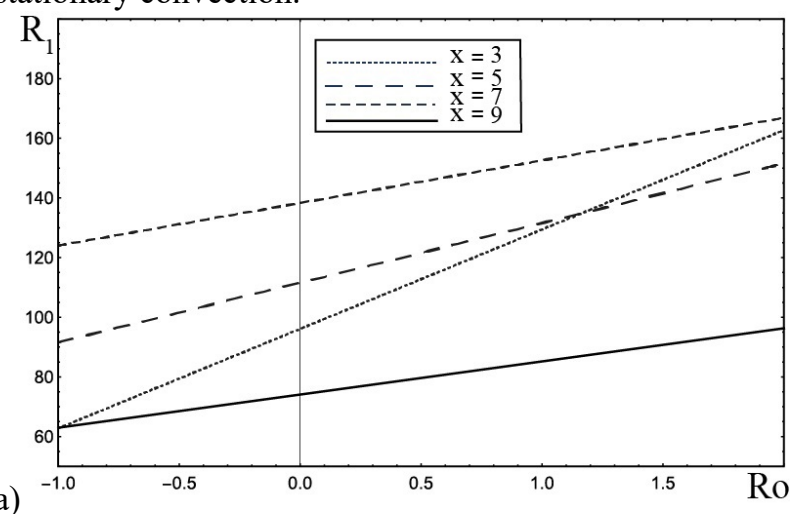

б)

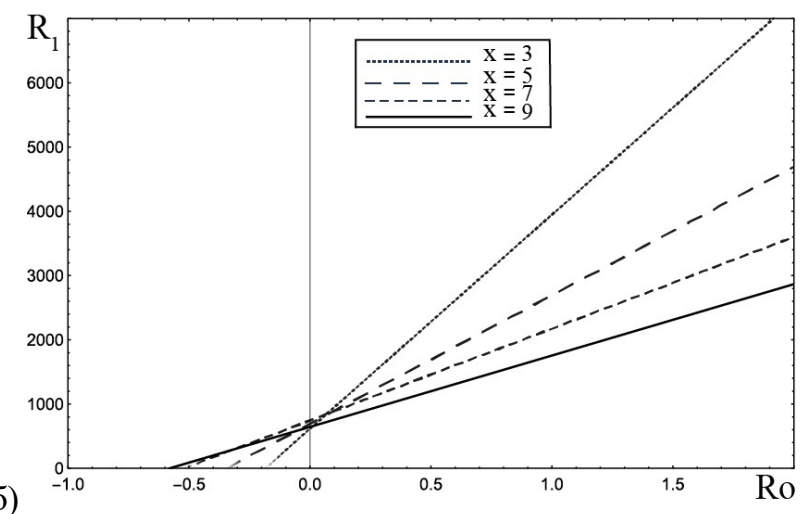

Fig. 8. Dependence of the Rayleigh number $R_{1}$ on the Rossby number $R o$ for various wave numbers $x=k / \pi$ with constant parameters: a) $Q_{1}=50, T_{1}=100, P m=1$; b) $Q_{1}=100, T_{1}=1000, P m=1$.

Without considering the thermal processes, i.e. when there is no preheating $R a=0$, from equation (72) we obtain the threshold value of the hydrodynamic Rossby number $R o$ for the standard MRI (SMRI) taking into account dissipative processes (see, for example, [29]): 


$$
R o_{c r}=-\frac{a^{2}\left(a^{4}+\pi^{2} H a^{2}\right)^{2}+\pi^{2} a^{4} T a}{\pi^{2} T a\left(a^{4}+\pi^{2} H a^{2} P m\right)}, \quad a^{2}=k^{2}+\pi^{2}
$$

When turning to the dimensional variables $\frac{\pi^{2} H a^{2}}{a^{4}} \rightarrow \frac{\omega_{A}^{2}}{\omega_{\nu} \omega_{\eta}}, \frac{\pi^{2} H a^{2} P m}{a^{4}} \rightarrow \frac{\omega_{A}^{2}}{\omega_{\eta}^{2}}, \frac{T a}{a^{4}} \rightarrow \frac{4 \Omega^{2}}{\omega_{v}^{2}}, \frac{\pi^{2}}{a^{2}} \rightarrow \xi^{2}$ we find the expression for $R o_{c r}$ [29]:

$$
R o_{c r}=-\frac{\left(\omega_{A}^{2}+\omega_{v} \omega_{\eta}\right)^{2}+4 \xi^{2} \Omega^{2} \omega_{\eta}^{2}}{4 \Omega^{2} \xi^{2}\left(\omega_{A}^{2}+\omega_{\eta}^{2}\right)}
$$

Therefore, in the extreme case, when $R a=0$, a magneto-rotational instability arises in the inhomogeneously rotating layer of the electrically conducting fluid in the continuous magnetic field.

Then, using solution (71) from equations (56) and (58) we define the expression for the perturbations of the magnetic field $H_{z}$ and temperature $\Theta$ through amplitude $W_{0}$ :

$$
H_{z}=\frac{\pi P m}{\operatorname{Pr}\left(\pi^{2}+k^{2}\right)} \cdot W_{0} \cos \pi z, \quad \Theta=\frac{W_{0}}{\pi^{2}+k^{2}} \cdot \sin \pi z
$$

These solutions satisfy the free boundary conditions of (59). Then we find the expressions for the components of the vortex $\xi$ and current $\zeta$ from equations (55) and (57), which, when using (56), take the form:

$$
\begin{aligned}
& \left(\widehat{D}^{2}-k^{2}\right) \zeta-\sqrt{T a}(1+R o) \operatorname{Pr} P^{-1}\left(\widehat{D}^{2}-k^{2}\right) H_{z}+\tilde{Q} \hat{D} \xi=0 \\
& \left(\widehat{D}^{2}-k^{2}\right) \xi+\operatorname{Pr}^{-1} \operatorname{Pm} \hat{D} \zeta-\sqrt{T a} \operatorname{PmRo} \hat{D} H_{z}=0
\end{aligned}
$$

Acting on equation (76) by operator $\widehat{D}^{2}-k^{2}$, and on equation (77) by operator $\tilde{Q} \hat{D}$, and then subtracting one equation from the other, we'll find the equation for the current $\zeta$ :

$$
\left[\left(\widehat{D}^{2}-k^{2}\right)^{2}-Q \widehat{D}^{2}\right] \zeta-\sqrt{T a}(1+R o) \operatorname{Pr} \operatorname{Pm}^{-1}\left(\widehat{D}^{2}-k^{2}\right)^{2} H_{z}+\sqrt{T a} Q \operatorname{Pr} R o \widehat{D}^{2} H_{z}=0
$$

Substituting the solution for $H_{z}$ from equation (75) into this equation we get:

$$
\zeta=\frac{\pi \sqrt{T a}(1+R o)\left(\pi^{2}+k^{2}\right)^{2}+\pi^{3} \sqrt{T a} Q P m R o}{\left(\pi^{2}+k^{2}\right)\left(\left(\pi^{2}+k^{2}\right)^{2}+\pi^{2} Q\right)} \cdot W_{0} \cos \pi z
$$

Now we proceed to the definition of the equation for the vortex component $\xi$ from equations (76)-(77). For this, we act on equation (76) by operator $\operatorname{Pr}^{-1} P m$, and on equation (77) by operator $\widehat{D}^{2}-k^{2}$, then subtracting one equation from the other, we find the equation for $\xi$ :

$$
\left[\left(\widehat{D}^{2}-k^{2}\right)^{2}-Q \widehat{D}^{2}\right] \xi+\sqrt{T a}(1+R o(1-P m))\left(\widehat{D}^{2}-k^{2}\right) \hat{D} H_{z}=0
$$

Similarly, for this equation too, using the solution for $H_{z}$ from (75), we obtain:

$$
\xi=-\frac{\pi^{2} \sqrt{T a}(1+R o(1-P m)) P m}{\left(\left(\pi^{2}+k^{2}\right)^{2}+\pi^{2} Q\right) \operatorname{Pr}} \cdot W_{0} \sin \pi z
$$

The obtained expressions (79) and (81), respectively, for the components of the vortex and of the current, satisfy the boundary conditions of (59).

\section{Topological characteristics of stationary solutions}

Note, that the stationary solutions obtained above for the hydrodynamic $(W, \xi)$ and magnetic $\left(H_{z}, \zeta\right)$ fields have a non-trivial topology. The average hydrodynamic helicity $\mathrm{H}_{g}=\int \vec{v} r o t \vec{v} d V$ has the meaning of a measure of 
"knotting" of the velocity field $\vec{v}$ and, similarly, the current helicity $\mathrm{H}_{c}=\int \vec{B} \operatorname{rot} \vec{B} d V$ is defined as a measure of "knotting" of the magnetic field force lines $\vec{B}$ [45]. We calculated the helicity of $\mathrm{H}_{g}$ and $\mathrm{H}_{c}$ for the deterministic fields (of the velocity and magnetic field) of stationary roll convection with averaging over the whole layer volume $(V)$. As the calculations show, the current helicity $\mathrm{H}_{c}$ has an opposite sign relative to the hydrodynamic helicity $\mathrm{H}_{g}$, and as a result, stabilizes the operation of the dynamo [46] due to generation of the hydrodynamic helicity by rotating the electrically conductive fluid.

The hydrodynamic helicity plays an important role in $\alpha$-effect onset, due to which large-scale magnetic and vortex fields are generated (see, for example, [6]). In the theory of turbulent dynamo [45] the mean helicity of the velocity field is the result of averaging over the ensemble of realizations of a random field in the given volume of fluid. Helicity naturally arises in the turbulence of a rotating body. The physical mechanism of the helicity origin is described in [47] by the example of the the Sun convective zone. In the northern hemisphere of the convective zone, the rising substance will expand and rotate under the action of Coriolis forces, resulting in a left-handed spiral movement. The sinking substance is compressed, and under the action of Coriolis forces is forced to rotate in the opposite direction, also making a left-handed spiral movement. It is obvious, that in the southern hemisphere the right-handed spiral movements will prevail. The uncompensated right-handed and left-handed movements lead to non-zero helicity. In other words, the properties of the turbulent velocity field are non-invariable with respect to the parity transformation, i.e. transition from the right coordinate system $(x, y, z)$ to the left one $(-x,-y,-z)$ (reflective non-invariance of the field $\vec{v}$ ). The $\alpha$-coefficient estimate values for the conditions of the Sun convective zone range from a few $\mathrm{cm} / \mathrm{s}$ to $10^{4} \mathrm{~cm} / \mathrm{s}$, what means a greater degree of uncertainty when transferring the results of the calculations for the dynamo models to the real solar conditions [48-49]. However, as the astronomical observations show [50], the Sun convective zone has an evident orderliness, i.e. a developed cellular structure of different scale. In [49] a numerical simulation of cell-like flows, which are similar to the really observed ones, were carried out, what allowed calculating directly the velocity field average helicity. The helicity was averaged in [49] over the volume, and not over the ensemble of realizations, as long as the velocity field in such a formulation of the problem is deterministic. The analysis of the helicity of such quasi-ordered convective flows can reduce the spread in the estimate values, which are used in the theory of the medium fields dynamo.

Thus, the results of the calculation of the average hydrodynamic and magnetic helicity of stationary fields give all grounds for the development of the theory of convective dynamo in a non-uniformly rotating conducting medium with the external magnetic field.

\section{WEAKLY NONLINEAR STAGE OF STATIONARY CONVECTION}

To describe the nonlinear convective phenomena in the inhomogeneously rotating layer of the electrically conducting fluid, it is convenient to turn from the cylindrical coordinate system $(R, \varphi, z)$ to the local Cartesian $(X, Y, Z)$ one. If we consider a fixed region of the fluid layer with a radius $R_{0}$ and angular velocity of rotation $\Omega_{0}=\Omega\left(R_{0}\right)$, then the coordinates $X=R-R_{0}$ correspond to the radial direction, $Y=R_{0}\left(\varphi-\varphi_{0}\right)$ - to azimuthal, and $Z=z$ - to vertical (see Fig. 9).

a)

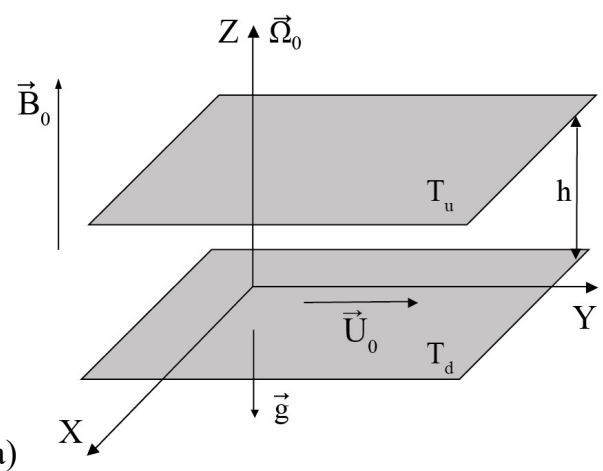

б)

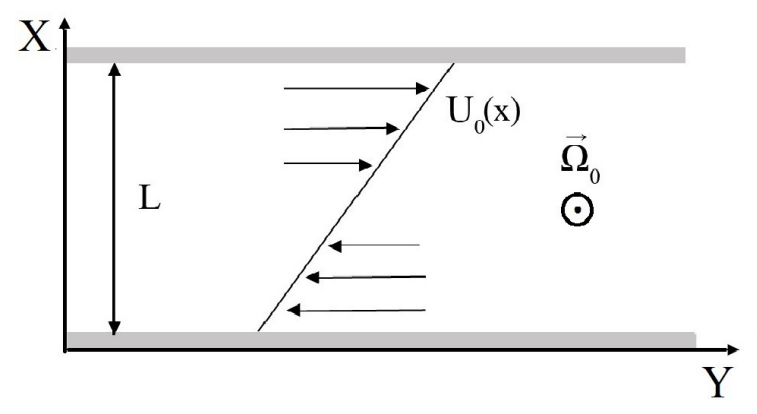

Fig. 9. a) Cartesian approximation of the problem for a non-uniformly rotating magnetic convection, inhomogeneous rotation in the local Cartesian coordinate system consisting of rotation with constant angular velocity $\vec{\Omega}_{0}$ and shearing velocity $U_{0} \| O Y$; b) Scheme of the shear flow in rotating flows, the flow being approximated in the local Cartesian coordinate system as a linear shift with velocity $U_{0}(X)$, with the value of the flow being limited by coordinates $X \in[0, L]$ and $Z \in[0, h]$, and by coordinate $Y$ being unlimited. 
In this case, the fluid layer inhomogeneous rotation can be represented locally as the rotation with the constant angular velocity $\vec{\Omega}_{0}$ and azimuthal shear [51], whose velocity profile is locally linear $\vec{U}_{0}=-q \Omega_{0} X \vec{e}_{y}$, where $q \equiv-d \ln \Omega / d \ln R$ is the dimensionless shear parameter, determined from the profile of the angular velocity of rotation $\Omega(R)=\Omega_{0}\left(R / R_{0}\right)^{-q}$. The shear parameter $q$ is bound up with the hydrodynamic Rossby number $R o=\frac{R}{2 \Omega} \frac{\partial \Omega}{\partial R}$ by the relation $q=-2 R o$. Note, that the accretion disks with a shear parameter $q=3 / 2$ $(R o=-3 / 4)$ correspond to the Keplerian disk, $q=2(R o=-1)$ corresponds to the disk with a constant angular momentum or the Rayleigh rotation profile. The case of $q=1 \quad(R o=-1 / 2)$ corresponds to the system with a flat rotation curve, and that of $q=0 \quad(R o=0)$ - to the homogeneous (or solid- state) rotation with a constant angular velocity.

The equations for the perturbations of the velocity $\vec{u}=(u, v, w)$ of the magnetic field $\vec{b}=(\tilde{u}, \tilde{v}, \tilde{w})$ and the temperature $\theta$ in the local Cartesian coordinate system take the following form:

$$
\begin{gathered}
\frac{\partial \vec{u}}{\partial t}-q \Omega_{0} X \frac{\partial u}{\partial Y}+(\vec{u} \nabla) \vec{U}_{0}+2 \vec{\Omega}_{0} \times \vec{u}+(\vec{u} \nabla) \vec{u}=-\frac{1}{\rho_{0}} \nabla \tilde{p}+\frac{1}{4 \pi \rho_{0}}\left(\left(\overrightarrow{B_{0}} \nabla\right) \vec{b}+(\vec{b} \nabla) \vec{b}\right)+g \beta \theta \vec{e}+v \nabla^{2} \vec{u} \\
\frac{\partial \vec{b}}{\partial t}-q \Omega_{0} X \frac{\partial \vec{b}}{\partial Y}-\left(\vec{B}_{0} \nabla\right) \vec{u}-(\vec{b} \nabla) \vec{U}_{0}+(\vec{u} \nabla) \vec{b}-(\vec{b} \nabla) \vec{u}=\eta \nabla^{2} \vec{b} \\
\frac{\partial \theta}{\partial t}-q \Omega_{0} X \frac{\partial \theta}{\partial Y}+(\vec{u} \nabla) T_{0}+(\vec{u} \nabla) \theta=\chi \nabla^{2} \theta \\
\operatorname{div} \vec{u}=0, \quad \operatorname{div} \vec{b}=0
\end{gathered}
$$

here the pressure $\tilde{p}$ includes the perturbed magnetic pressure $p_{m}=\frac{\vec{b}^{2}}{8 \pi}+\frac{\vec{b} \vec{B}_{0}}{4 \pi}: \tilde{p}=p+p_{m}$.

Like in the previous sections, we will consider the dynamics of axisymmetric perturbations, when all the perturbed quantities in equations (82) will depend only on two variables $(X, Z)$. In this case it is convenient to represent the vector fields $\vec{u}$ and $\vec{b}$ by scalar stream functions $\psi$ and $\phi$ :

$$
u=-\frac{\partial \psi}{\partial Z}, \quad w=\frac{\partial \psi}{\partial X}, \quad \tilde{u}=-\frac{\partial \varphi}{\partial Z}, \quad \tilde{w}=\frac{\partial \varphi}{\partial X}
$$

For convenience, in equations (82) we turn to the dimensionless variables, which we mark with an asterisk.

$$
(X, Z)=h\left(x^{*}, z^{*}\right), t=\frac{h^{2}}{v} t^{*}, \psi=\chi \psi^{*}, \phi=h B_{0} \phi^{*}, \quad v=\frac{\chi}{h} v^{*}, \tilde{v}=B_{0} \tilde{v}^{*}, \theta=A h \theta^{*},
$$

and performing some simple mathematical operations, we obtain a system of nonlinear equations for the inhomogeneously rotating magnetoconvection [36]:

$$
\begin{aligned}
& \left(\frac{\partial}{\partial t}-\nabla^{2} \psi\right) \nabla^{2} \psi+\sqrt{T a} \frac{\partial v}{\partial z}-\operatorname{Pr} \operatorname{Pm}^{-1} Q \frac{\partial}{\partial z} \nabla^{2} \phi-R a \frac{\partial \theta}{\partial x}=\operatorname{Pr} \operatorname{Pm}^{-1} Q \cdot J\left(\phi, \nabla^{2} \phi\right)-\operatorname{Pr}^{-1} \cdot J\left(\psi, \nabla^{2} \psi\right) \\
& \left(\frac{\partial}{\partial t}-\nabla^{2}\right) v-\sqrt{T a}(1+R o) \frac{\partial \psi}{\partial z}-\operatorname{Pr} \operatorname{Pm}^{-1} Q \frac{\partial \tilde{v}}{\partial z}=\operatorname{Pr} \operatorname{Pm}^{-1} Q \cdot J(\varphi, \tilde{v})-\operatorname{Pr}^{-1} \cdot J(\psi, v) \\
& \left.\left(\frac{\partial}{\partial t}-\operatorname{Pm}^{-1} \nabla^{2}\right) \phi-\operatorname{Pr}^{-1} \frac{\partial \psi}{\partial z}=-\operatorname{Pr}^{-1} J(\psi, \phi)\right) \\
& \left(\frac{\partial}{\partial t}-\operatorname{Pm}^{-1} \nabla^{2}\right) \tilde{v}-\operatorname{Pr}^{-1} \frac{\partial v}{\partial z}+\operatorname{Ro} \sqrt{T a} \frac{\partial \varphi}{\partial z}=\operatorname{Pr}^{-1}(J(\varphi, v)-J(\psi, \tilde{v})) \\
& \left(\frac{\partial}{\partial t}-\operatorname{Pr}^{-1} \nabla^{2}\right) \theta-\operatorname{Pr}^{-1} \frac{\partial \psi}{\partial x}=-\operatorname{Pr}^{-1} J(\psi, \theta)
\end{aligned}
$$


In equations (83) the asterisk is omitted. In the absence of the thermal phenomena, the system of equations (83) was used to study the nonlinear saturation mechanism of the standard MRI [52]. The system of equations (83) is complemented by the following boundary conditions:

$$
\begin{gathered}
\psi=\nabla^{2} \psi=0, \quad \frac{d v}{d z}=0, \quad \tilde{v}=0, \quad \frac{d \varphi}{d z}=0, \quad \theta=0 \quad \text { при } \quad z=0, \\
\psi=\nabla^{2} \psi=0, \quad \frac{d v}{d z}=0, \quad \tilde{v}=0, \quad \frac{d \varphi}{d z}=0, \quad \theta=0 \quad \text { при } \quad z=1 .
\end{gathered}
$$

Further, we apply the system of equations (83) and boundary conditions of (84) to study the weakly nonlinear convection mode.

\section{Equation of finite amplitude for the stationary convection}

The weakly nonlinear theory of convective instability describes the evolution of perturbations of not too high, but finite amplitude. The small amplitude of convective cells is superimposed on the main flow. If this amplitude is of the $O\left(\varepsilon^{1}\right)$ order, then the interaction of cells with one another leads to the second harmonic and to nonlinearity of the order $O\left(\varepsilon^{2}\right)$, and then to nonlinearity $O\left(\varepsilon^{3}\right)$, etc. Here the nonlinear terms in equations (83) are considered as a perturbed response for the linear convection problem. In this case the Rayleigh parameter $R a$, which controlls the convection is close to critical $R a_{c}$, i.e. to steady state of convection. The influence of the unstable modes is small, and therefore our task is to obtain equations describing the interaction of these modes. The general scheme for formulation of the weakly nonlinear theory is as follows. Since the small parameter of our problem is the relative deviation of the Rayleigh number $R a$ from the critical value $R a_{c}$ :

$$
\varepsilon^{2}=\frac{R a-R a_{c}}{R a_{c}} \ll 1,
$$

then all the perturbed values $V$ in the equations of the type $\hat{L} V=-N(V \mid V)$ are represented as a series in the perturbation theory:

$$
V \rightarrow \varepsilon V^{(1)}+\varepsilon^{2} V^{(2)}+\varepsilon^{3} V^{(3)}+\ldots,
$$

where $N(V \mid V)$ are the nonlinear terms.

The equations for the perturbations in various orders of $\varepsilon$ take the form:

$$
\begin{gathered}
\mathcal{E}^{1}: \widehat{L}^{(0)} V^{(1)}=0 \\
\varepsilon^{2}: \widehat{L}^{(0)} V^{(2)}=-N\left(V^{(1)} \mid V^{(1)}\right) \\
\varepsilon^{3}: \widehat{L}^{(0)} V^{(3)}=-\widehat{L}^{(2)} V^{(0)}-N\left(V^{(1)} \mid V^{(2)}\right)-N\left(V^{(2)} \mid V^{(1)}\right)
\end{gathered}
$$

The condition for solving this chain of nonlinear equations is known as Fredholm's alternative (see, for example, [53])

$$
\left\langle V^{\dagger}, R . H .\right\rangle=0
$$

Here $V^{\dagger}$ is a non-trivial solution of the linear self-adjoint problem $\widehat{L}^{\dagger} V^{\dagger}=0$, where $\widehat{L}^{\dagger}$ is a self-adjoint operator, which is determined from the following relation:

$$
\left\langle V^{\dagger}, \widehat{L} V\right\rangle \equiv\left\langle\hat{L}^{\dagger} V^{\dagger}, V\right\rangle
$$

where $\langle$,$\rangle is the inner product, which here has the following definition:$

$$
\langle f, g\rangle=\int_{z=0}^{1} \int_{x=0}^{2 \pi / k_{c}} f \cdot g d x d z,
$$

$R . H$. are right sides of the perturbed equations with nonlinear terms. We apply these general principles of solving nonlinear equations to our problem. For simplicity we will take into account the nonlinear terms in (83) only in the heat balance equation. As will be shown below (see Appendix), this approximation is equivalent to the application of the Galerkin approximation of the minimal order (118) to equations (83). We represent all the variables in equations (83) as an asymptotic expansion: 


$$
\begin{aligned}
& R a=R a_{c}+\varepsilon^{2} R a_{2}+\varepsilon^{4} R a_{4}+\ldots \\
& \psi=\varepsilon \psi_{1}+\varepsilon^{2} \psi_{2}+\varepsilon^{3} \psi_{3}+\ldots \\
& v=\varepsilon v_{1}+\varepsilon^{2} v_{2}+\varepsilon^{3} v_{3}+\ldots \\
& \phi=\varepsilon \phi_{1}+\varepsilon^{2} \phi_{2}+\varepsilon^{3} \phi_{3}+\ldots \\
& \tilde{v}=\varepsilon \tilde{v}_{1}+\varepsilon^{2} \tilde{v}_{2}+\varepsilon^{3} \tilde{v}_{3}+\ldots \\
& \theta=\varepsilon \theta_{1}+\varepsilon^{2} \theta_{2}+\varepsilon^{3} \theta_{3}+\ldots
\end{aligned}
$$

We assume that the amplitudes of the perturbed quantities depend only on the slow time $\tau=\varepsilon^{2} t$. Substituting the expansion of (87) into the system of equations (83), we solve it for different orders of $\mathcal{E}$. In the lowest order, we get the equation:

$$
\widehat{L} M_{1}=0
$$

where $M_{1}=\left[\begin{array}{c}\psi_{1} \\ v_{1} \\ \varphi_{1} \\ \tilde{v}_{1} \\ \theta_{1}\end{array}\right], \widehat{L}$ is the matrix operator of the form:

$$
\widehat{L}=\left[\begin{array}{ccccc}
-\nabla^{4} & \sqrt{T a} \frac{\partial}{\partial z} & -P m^{-1} \operatorname{Pr} Q \frac{\partial}{\partial z} \nabla^{2} & 0 & -R a_{c} \frac{\partial}{\partial x} \\
-\sqrt{T a}(1+R o) \frac{\partial}{\partial z} & -\nabla^{2} & 0 & -P m^{-1} \operatorname{Pr} Q \frac{\partial}{\partial z} & 0 \\
-\operatorname{Pr}^{-1} \frac{\partial}{\partial z} & 0 & -P m^{-1} \nabla^{2} & 0 & 0 \\
0 & -\operatorname{Pr}^{-1} \frac{\partial}{\partial z} & R o \sqrt{T a} \frac{\partial}{\partial z} & -P m^{-1} \nabla^{2} & 0 \\
-\operatorname{Pr}^{-1} \frac{\partial}{\partial x} & 0 & 0 & 0 & -\operatorname{Pr}^{-1} \nabla^{2}
\end{array}\right] .
$$

The solutions of the system of equations (88) with the boundary conditions of (84) have, respectively, the form:

$\psi_{1}=A(\tau) \sin k_{c} x \sin \pi z, \theta_{1}=\frac{A(\tau) k_{c}}{a^{2}} \cos k_{c} x \sin \pi z, \phi_{1}=\frac{A(\tau) \pi P m}{a^{2} \operatorname{Pr}} \sin k_{c} x \cos \pi z$,

$\tilde{v}_{1}=-\frac{A(\tau) \pi^{2} \sqrt{T a}(1+R o(1-P m)) P m}{\operatorname{Pr}\left(a^{4}+\pi^{2} Q\right)} \sin k_{c} x \sin \pi z$,

$v_{1}=\frac{A(\tau) \pi \sqrt{T a}}{a^{2}} \cdot \frac{(1+R o) a^{4}+\pi^{2} Q P m R o}{a^{4}+\pi^{2} Q} \sin k_{c} x \cos \pi z, \quad a^{2}=k_{c}^{2}+\pi^{2}$.

The amplitude $A(\tau)$ is still unknown. The critical value of the Rayleigh number $R a_{c}$ for the stationary magnetoconvection in a non-uniformly rotating electrically conducting medium is found from the first equation of the system (88) and has the form of formula (72) obtained in the linear theory:

$$
R a_{c}=\frac{\left(\pi^{2}+k_{c}^{2}\right)^{3}}{k_{c}^{2}}+\frac{\pi^{2}\left(\pi^{2}+k_{c}^{2}\right) Q}{k_{c}^{2}}+\frac{\pi^{2}\left(\pi^{2}+k_{c}^{2}\right)^{2} T a}{k_{c}^{2}\left(\left(\pi^{2}+k_{c}^{2}\right)^{2}+\pi^{2} Q\right)}+\frac{\pi^{2} T a R o\left(\left(\pi^{2}+k_{c}^{2}\right)^{2}+\pi^{2} Q P m\right)}{k_{c}^{2}\left(\left(\pi^{2}+k_{c}^{2}\right)^{2}+\pi^{2} Q\right)} .
$$


For the second order of $\mathcal{E}$, we have the following equation:

$$
\widehat{L} M_{2}=N_{2} \text {, }
$$

where $M_{2}=\left[\begin{array}{c}\psi_{2} \\ v_{2} \\ \varphi_{2} \\ \tilde{v}_{2} \\ \theta_{2}\end{array}\right], \quad N_{2}=\left[\begin{array}{c}N_{21} \\ N_{22} \\ N_{23} \\ N_{24} \\ N_{25}\end{array}\right] ; \quad N_{21}=N_{22}=N_{23}=N_{24}=0$

$$
N_{25}=-\operatorname{Pr}^{-1}\left[\frac{\partial \psi_{1}}{\partial x} \frac{\partial \theta_{1}}{\partial z}-\frac{\partial \theta_{1}}{\partial x} \frac{\partial \psi_{1}}{\partial z}\right]
$$

Using solutions of (89) and boundary conditions of (84), we find solutions to equations (90):

$$
\psi_{2}=0, \quad \theta_{2}=-\frac{A^{2}(\tau) k_{c}^{2}}{8 \pi a^{2}} \sin (2 \pi z), \quad \phi_{2}=0, \quad v_{2}=0, \tilde{v}_{2}=0 .
$$

To analyze the intensity of the heat transfer, a horizontally-averaged heat flux is introduced at the boundary of the layer of electrically conducting fluid (Nusselt number):

$$
N u(\tau)=1+\frac{\left[\frac{k_{c}}{2 \pi} \int_{0}^{2 \pi / k_{c}}\left(\frac{\partial \theta_{2}}{\partial z}\right) d x\right]_{z=0}}{\left[\frac{k_{c}}{2 \pi} \int_{0}^{2 \pi / k_{c}}\left(\frac{\partial T_{0}}{\partial z}\right) d x\right]_{z=0}}=1+\frac{k_{c}^{2}}{4 a^{2}} A^{2}(\tau)
$$

The heat flow intensity (of Nusselt number) will be analyzed after the expression for the amplitude $A(\tau)$ is obtained.

For the third order of $\varepsilon$ we find:

$$
\widehat{L} M_{3}=N_{3}
$$

where $M_{3}=\left[\begin{array}{c}\psi_{3} \\ v_{3} \\ \varphi_{3} \\ \tilde{v}_{3} \\ \theta_{3}\end{array}\right], N_{3}=\left[\begin{array}{c}N_{31} \\ N_{32} \\ N_{33} \\ N_{34} \\ N_{35}\end{array}\right] ; N_{31}=-\frac{\partial}{\partial \tau} \nabla^{2} \psi_{1}+R a_{2} \frac{\partial \theta_{1}}{\partial x}=\left(a^{2} \frac{\partial A(\tau)}{\partial \tau}-R a_{2} \frac{k_{c}^{2} A(\tau)}{a^{2}}\right) \sin k_{c} x \sin \pi z$

$N_{32}=-\frac{\partial v_{1}}{\partial \tau}=-\frac{\pi \sqrt{T a}}{a^{2}} \cdot \frac{(1+R o) a^{4}+\pi^{2} Q P m R o}{a^{4}+\pi^{2} Q} \cdot \frac{\partial A(\tau)}{\partial \tau} \sin k_{c} x \cos \pi z$

$N_{33}=-\frac{\partial \phi_{1}}{\partial \tau}=-\frac{\pi P m}{a^{2} \operatorname{Pr}} \cdot \frac{\partial A(\tau)}{\partial \tau} \sin k_{c} x \cos \pi z$

$N_{34}=-\frac{\partial \tilde{v}_{1}}{\partial \tau}=\frac{\pi^{2} \sqrt{T a}(1+R o(1-P m)) P m}{\operatorname{Pr}\left(a^{4}+\pi^{2} Q\right)} \cdot \frac{\partial A(\tau)}{\partial \tau} \sin k_{c} x \sin \pi z$,

$N_{35}=-\frac{\partial \theta_{1}}{\partial \tau}-\operatorname{Pr}^{-1}\left[\frac{\partial \psi_{1}}{\partial x} \frac{\partial \theta_{2}}{\partial z}-\frac{\partial \theta_{2}}{\partial x} \frac{\partial \psi_{1}}{\partial z}+\frac{\partial \psi_{2}}{\partial x} \frac{\partial \theta_{1}}{\partial z}-\frac{\partial \theta_{1}}{\partial x} \frac{\partial \psi_{2}}{\partial z}\right]=$

$=-\frac{k_{c}}{a^{2}} \frac{\partial A(\tau)}{\partial \tau} \cos k_{c} x \sin \pi z+\frac{\operatorname{Pr}^{-1} k_{c}^{3}}{4 a^{2}} A^{3}(\tau) \cos k_{c} x \sin \pi z \cos 2 \pi z$. 
The solvability condition (an alternative of Fredholm) for the equations of the third order $O\left(\varepsilon^{3}\right)$ is found from equation (85):

$$
\int_{z=0}^{1} \int_{x=0}^{2 \pi / k}\left[\hat{P} \psi_{1}^{\dagger} \cdot R_{31}+R a_{c} \hat{P} \theta_{1}^{\dagger} \cdot R_{32}+Q P r^{2} P m^{-1} \widehat{P} \nabla^{2} \phi_{1}^{\dagger} \cdot R_{33}+v_{1}^{\dagger} \cdot R_{34}\right] d x d z=0
$$

where indcations are introduced

$$
\begin{gathered}
\widehat{P}=(1+R o) \nabla^{4}-Q \operatorname{PmRo} \frac{\partial^{2}}{\partial z^{2}}, R_{31}=N_{31}, R_{32}=N_{35}, R_{33}=N_{33}, \\
R_{34}=-\nabla^{4} N_{32}+\operatorname{QPr} \frac{\partial}{\partial z} \nabla^{2} N_{34}+\sqrt{\operatorname{Ta}} Q \operatorname{PmPrRo} \frac{\partial^{2} N_{33}}{\partial z^{2}}
\end{gathered}
$$

The expressions for $\psi_{1}^{\dagger}, \theta_{1}^{\dagger}, \phi_{1}^{\dagger}, v_{1}^{\dagger}$ are determined from the solution of the linear self-adjoint problem $\widehat{L}^{\dagger} M_{1}^{\dagger}=0$ :

$$
\begin{gathered}
\psi_{1}^{\dagger}=A(\tau) \sin k_{c} x \sin \pi z, \theta_{1}^{\dagger}=-\frac{A(\tau) k_{c}}{a^{2}} \cos k_{c} x \sin \pi z, \quad \phi_{1}^{\dagger}=-\frac{A(\tau) \pi P m}{a^{2} \operatorname{Pr} \sin k_{c} x \cos \pi z,} \\
v_{1}^{\dagger}=-\frac{A(\tau) \pi \sqrt{T a}}{a^{2}} \cdot \frac{(1+R o) a^{4}+\pi^{2} Q P m R o}{a^{4}+\pi^{2} Q} \sin k_{c} x \cos \pi z
\end{gathered}
$$

The matrix $M_{1}^{\dagger}$ has the form $M_{1}^{\dagger}=\left(\psi_{1}^{\dagger}, \theta_{1}^{\dagger}, \phi_{1}^{\dagger}, v_{1}^{\dagger}\right)^{T r}$ and $\hat{L}^{\dagger}$ is a self-adjoint matrix operator:

$$
\hat{L}^{\dagger}=\left[\begin{array}{cccc}
-\nabla^{4} & R a_{c} \hat{P} \frac{\partial}{\partial x} & P m^{-1} \operatorname{Pr} Q \widehat{P} \frac{\partial}{\partial z} \nabla^{2} & -\sqrt{T a} \hat{P} \frac{\partial}{\partial z} \\
R a_{c} \hat{P} \frac{\partial}{\partial x} & -R a_{c} \hat{P} \nabla^{2} & 0 & 0 \\
P m^{-1} \operatorname{Pr} Q \hat{P} \frac{\partial}{\partial z} \nabla^{2} & 0 & -Q \operatorname{Pr} P m^{-2} \widehat{P} \nabla^{4} & 0 \\
-\sqrt{T a} \hat{P} \frac{\partial}{\partial z} & 0 & 0 & \left(\nabla^{4}-Q \frac{\partial^{2}}{\partial z^{2}}\right) \nabla^{2}
\end{array}\right]
$$

When performing integration in (94), we obtain a nonlinear equation for the amplitude $A(\tau)$, which refers to the Ginzburg-Landau equation or the Bernoulli differential equation with constant coefficients:

$$
A_{1} \frac{\partial A}{\partial \tau}-A_{2} A+A_{3} A^{3}=0
$$

Here the constant coefficients $A_{1,2,3}$ have the form:

$$
\begin{gathered}
A_{1}=\frac{a^{2}}{\operatorname{Pr}}+\frac{k_{c}^{2} R a_{c}}{a^{4}}-\frac{\pi^{2} Q P m}{a^{2} \operatorname{Pr}}-\frac{\pi^{2} T a\left((1+R o) a^{4}+\pi^{2} Q P m(R o P m-1)\right)}{\left(a^{4}+\pi^{2} Q\right)^{2} \operatorname{Pr}}-\frac{\pi^{4} T a R o Q P m^{2}}{a^{4}\left(a^{4}+\pi^{2} Q\right) \operatorname{Pr}} \\
A_{2}=\frac{k_{c}^{2} R a_{2}}{a^{2} \operatorname{Pr}}, \quad A_{3}=\frac{k_{c}^{4} R a_{c}}{8 a^{4} \operatorname{Pr}}
\end{gathered}
$$

The equation of the form (96) was obtained in many papers, where the weakly nonlinear mode of stationary convection was studied (see, for example, [14-20]). In contrast to these works, in our result (96) the non-uniform 
rotation $(R o \neq 0)$ of the electrically conducting fluid was taken into account. The analytical solution of equation (96) with a known initial condition $A_{0}=A(0)$ can be obtained using the Lagrange method (variations of the constant):

$$
A(\tau)=\frac{A_{0}}{\sqrt{\frac{A_{3}}{A_{2}} A_{0}^{2}+\left(1-A_{0}^{2} \frac{A_{3}}{A_{2}}\right) \exp \left(-\frac{2 \tau A_{2}}{A_{1}}\right)}}
$$

Assuming the initial amplitude to be equal to $A_{0}=0.7$ and $R a_{2} \approx R a_{c}$, by using the solution of (98), we can determine the variation in the heat transfer (of Nusselt number $N u$ ) with time $\tau$. The diagram of dependency $N u(\tau)$ is presented in Fig. 10. The diagram clearly shows the establishment of the final value $N u(\tau)$ due to the relationship between the number $N u(\tau)$ and the amplitude $A(\tau)$ (see equation (92)). The excess of number $N u$ over the unit is caused by the convection occurrence. Fig. 10 shows that in the course of time the heat transfer intensity proceeds most smoothly for the case of the non-uniform rotation with a negative profile $(R o<0)$. Fig. 11 presents the diagram of the Nusselt number $N u$ versus the Rossby number $R o$. Here we see (curves 1-4) that the heat transfer intensity increases in the direction of positive Rossby numbers $(R o>0)$.

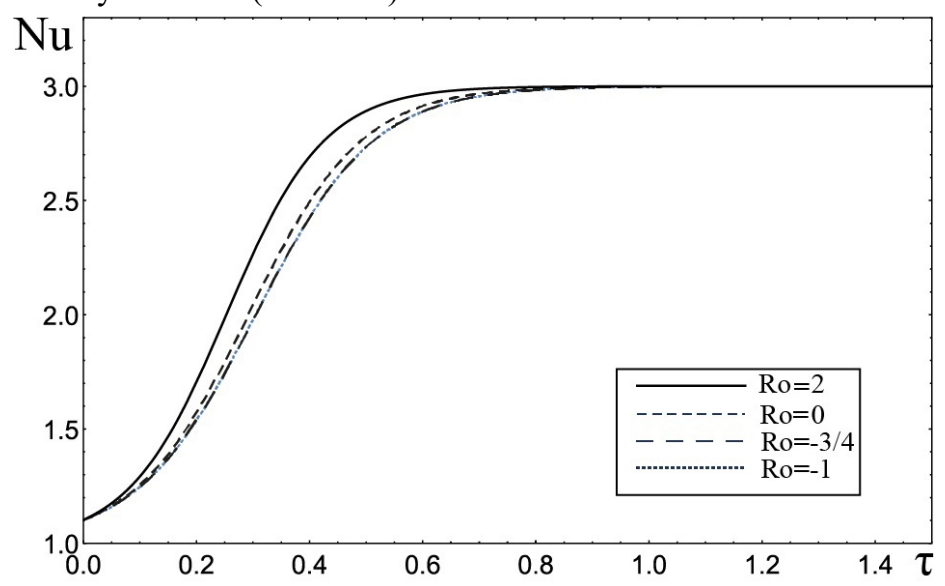

Fig. 10. The dependence of the Nusselt number $N u$ on time $\tau$ for different values of Rossby numbers $R o$ with constant parameters: $Q_{1}=80, T_{1}=10^{5}, R_{1}=9500, \operatorname{Pm}=1, \operatorname{Pr}=10$.

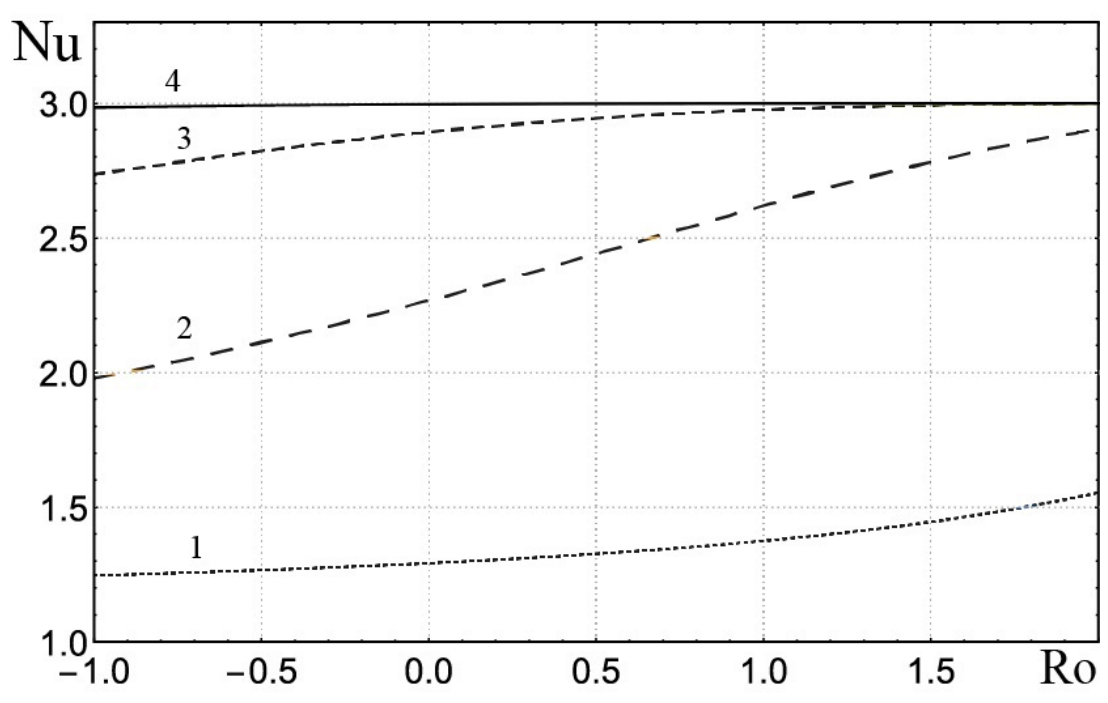

Fig. 11. Dependence of the Nusselt number $N u$ on the Rossby numbers $R o$ with constant parameters: $Q_{1}=80, T_{1}=10^{5}$, $R_{1}=9500, \operatorname{Pm}=1, \operatorname{Pr}=10 ; 1,2,3,4$ curves correspond to times $\tau=0.1,0.3,0.5,1$. 


\section{CONCLUSION}

Applying the Lyenard-Shepard algorithm, we obtained the stability criteria for the inhomogeneously rotating plasma in the axially uniform magnetic field with a vertical temperature gradient under the condition of weak stratification. This problem was also investigated for thin rotating layers of the magnetized fluid with different temperatures on the layer surfaces (the Rayleigh-Benard convection).

A linear and weakly nonlinear theory of stationary convection in the non-uniformly rotating electrically conducting fluid in the axial magnetic field was developed. In the linear approximation, or in the first order in the small parameter of supercriticality of the Rayleigh number $\mathcal{E}=\sqrt{\left(R a-R a_{c}\right) / R a_{c}}$, we obtained an expression for the critical Rayleigh number $R a_{c}$, which in the limit of the uniform rotation $R o=0$ coincides with the known results of Chandrasekhar [1]. In the absence of the temperature gradient $(R a=0)$ we obtaine the known criterion for MRI occurrence [29]. The case of the negative profile of the inhomogeneous rotation $R o<0$ leads to some decrease in the instability threshold, and thus has a destabilizing effect on the instability development. And on the contrary, the positive rotation profiles $R o>0$ increase the critical value of the Rayleigh number, thus making a stabilizing effect on the convection development. In the third order of $\mathcal{E}$, we obtained the nonlinear Ginzburg-Landau equation, describing the evolution of the perturbation amplitude $A(\tau)$. The analysis of this equation solution has shown, that the flow intensity increases with the medium rotation at the positive Rossby numbers $R o>0$. In addition, it is shown that the weakly nonlinear convection, based on the equations of the six-mode $(6 D)$ Lorentz model from [36], is transformed into the identical Ginzburg-Landau equation.

In conclusion, we note, that the helicity properties of the stationary fields (vortex and magnetic) create preconditions for the development of the theory of convective dynamo in the rotating conducting medium with the external magnetic field and shear flow.

APPENDIX

\section{DERIVATION OF GINZBURG-LANDAU EQUATION FROM 6D-LAURENTZ EQUATIONS}

In [36] the nonlinear system of equations (83) was solved by the Galerkin method utilizing the minimal order expansion in $x$ and $z$-directions for the values $\psi, \phi, v, \tilde{v}$ and $\theta$ :

$$
\begin{aligned}
& \psi(x, z, t)=A(t) \sin (k x) \sin (\pi z), \\
& v=E(t) \sin (k x) \cos (\pi z), \\
& \phi(x, z, t)=B(t) \sin (k x) \cos (\pi z), \\
& \tilde{v}=F(t) \sin (k x) \sin (\pi z), \\
& \theta(x, y, t)=C(t) \cos (k x) \sin (\pi z)+D(t) \sin (2 \pi z),
\end{aligned}
$$

where $k=2 \pi h / L$ is the dimensionless wave number, $L$ is the typical layer length in the horizontal direction, and $A$, $B, C, D, E, F$ are the amplitudes of the perturbations. As a result of expansion of (99) substitution into equations (83), with the properties of the functions orthogonality taken into account, we obtain the Lorentz equations for the sixdimensional $(6 D)$ phase space [36]:

$$
\left\{\begin{array}{l}
\dot{X}=-X+R Y-T V-H U \\
\dot{V}=-V+H W+\sqrt{T a}(1+R o) X \\
\dot{U}=-P m^{-1} U+\operatorname{Pr}^{-1} X \\
\dot{W}=-P^{-1} W-P^{-1} V+R o \sqrt{T a} U \\
\dot{Y}=\operatorname{Pr}^{-1}(-Y+X-X Z) \\
\dot{Z}=\operatorname{Pr}^{-1}(-\gamma Z+X Y)
\end{array}\right.
$$

where a dot above denotes the time differentiation $\tilde{t}=a^{2} t$. For convenience, the following indications are introduced into (100): 


$$
R=\frac{k^{2} R a}{a^{6}}, \quad T=\frac{\pi^{2} \sqrt{T a}}{a^{6}}, \quad H=\frac{\pi^{2}}{a^{4}} \frac{Q P r}{P m}, \quad \gamma=\frac{4 \pi^{2}}{a^{2}}
$$

and amplitudes $A, B, C, D, E, F$ were rescaled in the form:

$$
\begin{gathered}
X(\tilde{t})=\frac{k \pi}{a^{2} \sqrt{2}} A(\tilde{t}), V(\tilde{t})=\frac{k E(\tilde{t})}{\sqrt{2}}, U(\tilde{t})=\frac{k B(\tilde{t})}{\sqrt{2}}, W(\tilde{t})=\frac{a^{2} k}{\pi \sqrt{2}} F(\tilde{t}), \\
Y(\tilde{t})=\frac{\pi C(\tilde{t})}{\sqrt{2}}, Z(\tilde{t})=-\pi D(\tilde{t}) .
\end{gathered}
$$

Following the method of [54], we represent all the perturbed values in equations (100) as an expansion into a series in the small supercriticality parameter of $\mathcal{E}$ :

$$
\begin{gathered}
\mathrm{X}=\varepsilon X_{1}+\varepsilon^{2} X_{2}+\varepsilon^{3} X_{3}+\ldots, \quad \mathrm{X}=[X, V, U, W, Y, Z]^{T r} \\
R=R_{0}+\varepsilon^{2} R_{2}+\ldots
\end{gathered}
$$

The amplitudes of the perturbed quantities depend only on the slow time $\tilde{\tau}=\varepsilon^{2} \tilde{t}$. For the first order of $\varepsilon$, after substituting expansion (101) into (100), we obtain a linear system of equations:

$$
L \mathrm{X}_{1}=0, \mathrm{X}_{1}=\left[X_{1}, V_{1}, U_{1}, W_{1}, Y_{1}, Z_{1}\right]^{T r},
$$

where $L$ matrix has the form:

$$
L=\left[\begin{array}{cccccc}
-1 & -T & -H & 0 & R_{0} & 0 \\
\sqrt{T a}(1+R o) & -1 & 0 & H & 0 & 0 \\
\operatorname{Pr}^{-1} & 0 & -P m^{-1} & 0 & 0 & 0 \\
0 & -\operatorname{Pr}^{-1} & R o \sqrt{T a} & -P m^{-1} & 0 & 0 \\
-\operatorname{Pr}^{-1} & 0 & 0 & 0 & -\operatorname{Pr}^{-1} & 0 \\
0 & 0 & 0 & 0 & 0 & -\gamma \operatorname{Pr}^{-1}
\end{array}\right] .
$$

The solutions of equations (102) have, respectively the form:

$$
\mathrm{X}_{1}=\left[X_{1}, \frac{\sqrt{T a}\left((1+R o) \operatorname{Pr}+H R o P m^{2}\right)}{\operatorname{Pr}+H P m} X_{1}, \frac{P m}{P r} X_{1},-\frac{\sqrt{T a} P m(1+R o(1-P m))}{\operatorname{Pr}+H P m} X_{1}, X_{1}, 0\right]^{T r}
$$

For the second order of $\mathcal{E}$, we have the following equation:

$$
L \mathrm{X}_{2}=\left[\mathfrak{R}_{21}, \Re_{22}, \Re_{23}, \Re_{24}, \Re_{25}, \Re_{26}\right]^{T r}, \mathrm{X}_{2}=\left[X_{2}, V_{2}, U_{2}, W_{2}, Y_{2}, Z_{2}\right]^{T r},
$$

where the nonlinear terms have, respectively, the form:

$$
\mathfrak{R}_{21}=0, \Re_{22}=0, \Re_{23}=0, \Re_{24}=0, \Re_{25}=-\operatorname{Pr}^{-1} X_{1} Z_{1}, \Re_{26}=\operatorname{Pr}^{-1} X_{1} Y_{1}
$$

The solutions of equations (104) have the form:

$$
\mathrm{X}_{2}=\left[X_{2}, \frac{\sqrt{T a}\left((1+R o) \operatorname{Pr}+H R o P m^{2}\right)}{\operatorname{Pr}+H P m} X_{2}, \frac{P m}{P r} X_{2},-\frac{\sqrt{T a} P m(1+R o(1-P m))}{\operatorname{Pr}+H P m} X_{2}, X_{2}, \frac{1}{\gamma} X_{1}^{2}\right]^{T r}
$$

Next we turn to the equations of the third order of $\mathcal{E}$ :

$$
L \mathrm{X}_{3}=\left[\Re_{31}, \Re_{32}, \Re_{33}, \Re_{34}, \Re_{35}, \Re_{36}\right]^{T r}, \mathrm{X}_{3}=\left[X_{3}, V_{3}, U_{3}, W_{3}, Y_{3}, Z_{3}\right]^{T r},
$$

where the nonlinear terms have, respectively, the form: 


$$
\begin{gathered}
\Re_{31}=-\frac{\partial X_{1}}{\partial \tilde{\tau}}+R_{2} Y_{1}, \Re_{32}=-\frac{\partial V_{1}}{\partial \tilde{\tau}}, \Re_{33}=-\frac{\partial U_{1}}{\partial \tilde{\tau}}, \Re_{34}=-\frac{\partial W_{1}}{\partial \tilde{\tau}}, \\
\Re_{35}=-\operatorname{Pr}^{-1}\left(X_{1} Z_{2}+X_{2} Z_{1}\right), \Re_{36}=-\frac{\partial Z_{1}}{\partial \tilde{\tau}}+\operatorname{Pr}^{-1}\left(X_{1} Y_{2}+X_{2} Y_{1}\right) .
\end{gathered}
$$

The condition of solvability (Fredholm alternative) of the nonlinear equations (106) in the third order of $\mathcal{E}$, according to the definition in (91), has the following form:

$$
\sum_{j=1}^{5} \Lambda_{3 j} \mathrm{X}_{1}^{\dagger}=0
$$

where

$$
\begin{gathered}
\Lambda_{31}=\Pi \operatorname{Pr}^{-1}\left(\frac{\partial X_{1}}{\partial \tau}-R_{2} Y_{1}\right), \Pi=\sqrt{T a}\left(1+R o+H R o \frac{P m^{2}}{P r}\right), \\
\Lambda_{32}=\Pi R_{0} \frac{\partial Y_{1}}{\partial \tilde{\tau}}+\Pi R_{0} \operatorname{Pr}^{-1}\left(X_{1} Z_{2}+X_{2} Z_{1}\right), \Lambda_{33}=-\frac{\partial Z_{1}}{\partial \tilde{\tau}}+\operatorname{Pr}^{-1}\left(X_{1} Y_{2}+X_{2} Y_{1}\right), \\
\Lambda_{34}=-H \Pi \frac{\partial U_{1}}{\partial \tilde{\tau}}, \Lambda_{35}=-T \operatorname{Pr}^{-1} \frac{\partial V_{1}}{\partial \tilde{\tau}}-T H P m \operatorname{Pr}^{-1} \frac{\partial W_{1}}{\partial \tilde{\tau}}-T H R o P m^{2} \sqrt{T a} \operatorname{Pr}^{-1} \frac{\partial U_{1}}{\partial \tilde{\tau}} .
\end{gathered}
$$

The elements of the matrix $\mathrm{X}_{1}^{\dagger}=\left[X_{1}^{\dagger}, Y_{1}^{\dagger}, Z_{1}^{\dagger}, U_{1}^{\dagger}, V_{1}^{\dagger}\right]^{T r}$ are the solutions of the linear self-adjoint problem $L^{\dagger} \mathrm{X}_{1}^{\dagger}=0$, where the self-adjoint matrix $L^{\dagger}$ is determined as:

$$
L^{\dagger}=\left[\begin{array}{ccccc}
\Pi \operatorname{Pr}^{-1} & -R_{0} \Pi \operatorname{Pr}^{-1} & 0 & H \Pi \operatorname{Pr}^{-1} & T \Pi \operatorname{Pr}^{-1} \\
-R_{0} \Pi \operatorname{Pr}^{-1} & R_{0} \Pi \operatorname{Pr}^{-1} & 0 & 0 & 0 \\
0 & 0 & -\gamma \operatorname{Pr}^{-1} & 0 & 0 \\
H \Pi \operatorname{Pr}^{-1} & 0 & 0 & -P m^{-1} \Pi H & 0 \\
T \Pi \operatorname{Pr}^{-1} & 0 & 0 & 0 & -\left(1+H P m \operatorname{Pr}^{-1}\right) T \operatorname{Pr}^{-1}
\end{array}\right]
$$

From equation (107) we get a non-linear equation for amplitude $A(\tau)$, here the equation completely coincides with the Ginzburg-Landau equation (96):

$$
A_{1} \frac{\partial A}{\partial \tau}-A_{2} A+A_{3} A^{3}=0
$$

Here the type of coefficients $A_{1,2,3}$ completely coincides with expressions (97). When deriving equation (108), we utilised the relationship of the rescaled derivative over the slow time: $\tilde{\tau}: \frac{\partial}{\partial \tilde{\tau}}=\frac{1}{a^{2}} \frac{\partial}{\partial \tau}$.

Thus, applying the asymptotic expansion of the perturbation theory to the $(6 D)$ Lorentz equations (100), we obtained the Ginzburg-Landau equation, which is identical to equation (96) obtained in the weakly non-linear theory for the finite amplitude.

\section{ORCID IDs}

Michael Kopp (D) https://orcid.org/0000-0001-7457-3272, Anatoly Tur (D) https://orcid.org/0000-0002-3889-8130, Volodymyr Yanovsky (D) https://orcid.org/0000-0003-0461-749X

\section{REFERENCES}

[1]. S. Chandrasekhar, Hydrodynamics and Hydromagnetic Stability (Oxford Uni. Press, London, 1961), p. 652.

[2]. G.Z. Gershuni and E.M. Zhukhovitckii, Convective Stability of Incompressible Fluids (Nauka, Moscow, 1972), p. 392 (in Russian)

[3]. A.V. Getling, Rayleigh-Benard Convection: Structures and Dynamics (URSS, Moscow, 1999), p. 235. (in Russian)

[4]. Chris A. Jones, Dynamo theory (University of Leeds, UK, 2007).

[5]. P.H. Roberts and G.A. Glatzmaier, Geophys. Astrophys. Fluid Dynam. 94(1), 47-84 (2001).

[6]. A. Tur and V.Yanovsky, Coherent Vortex Structures in Fluids and Plasmas (Springer, New York, 2017). p. 253.

[7]. I.A. Eltayeb, Proc. R. Soc. Lond. A. 326, 229-254 (1972). 
[8]. I.A. Eltayeb, J. Fluid Mech. 71(1), 161-179 (1975), doi:10.1017/S0022112075002480.

[9]. R. Avila and A. Cabello, Mathematical Problems in Engineering, 2013, 1-15 (2013), doi:10.1155/2013/236901.

[10]. W.V.R. Malkus and G. Veronis, J. Fluid Mech. 4(3), 225-260 (1958), doi:10.1017/S0022112058000410.

[11]. J.K. Bhattacharjee, J. Phy. A: Math. Gen. 22(24), L1135-L1189 (1989), doi:10.1088/0305-4470/22/24/001.

[12]. J.K. Bhattacharjee, Phy. Rev. A. 41, 5491-5494 (1990), doi:10.1103/PhysRevA.41.5491.

[13]. B.S., Bhadauria and P. Kiran, Ain Shams Eng. J. 5(4), 1287-1297 (2015), doi:10.1016/j.asej.2014.05.005.

[14]. R. Ramya, E.J. Shelin and G.K. Sangeetha, International Journal of Mathematics Trends and Technology, 54(6), 477-484 (2018), doi:10.14445/22315373/IJMTT-V54P558

[15]. P. Kiran, Ain Shams Eng. J. 7(2), 639-651 (2016), doi:10.1016/j.asej.2015.06.005

[16]. P.G. Siddheshwar, B.S. Bhadauria and A. Srivastava, Transp. Porous Media, 91(2), 585- 604 (2012), doi:10.1007/s11242-0119861-3

[17]. B.S. Bhadauria, P.G. Siddheshwar, J. Kumar and O.P. Suthar, Trans. Porous Med. 73(3), 633-647 (2012), doi:10.1007/s11242011-9925-4

[18]. P.G. Siddheshwar, B.S. Bhadauria, Pankaj Mishra and A.K. Srivastava, Int. J. Non Linear Mech. 47, 418-425 (2012), doi:10.1016/j.jinonlinmec.2011.06.006.

[19]. B.S. Bhadauria and P. Kiran, J. Appl. Fluid Mech. 8(4), 735-746 (2015), doi:10.18869/acadpub.jafm.73.238.22740.

[20]. B.S. Bhadauria and P. Kiran, Transp. Porous Media. 100, 279-295 (2013), doi:10.1007/s11242-013-0216-0.

[21]. B.S. Bhadauria, P. Kiran, Phys. Scr. 89(9), 095209 (2014), doi:10.1088/0031-8949/89/9/095209.

[22]. S. Aniss, M. Belhaq and M. Souhar, J. Heat Transfer, 123(3), 428-433 (2001), doi:10.1115/1.1370501.

[23]. S. Chandrasekhar, On the stability of the simplest solution of the equations of hydromagnetics. // Proc. Natl Acad. Sci. USA, 42(5), 273-276 (1956), doi:10.1073/pnas.42.5.273

[24]. E.P. Velikhov, Stability of an ideally conducting fluid flowing between cylinders rotating in a magnetic field, JETP, 36, 13981404 (1959). (in Russian)

[25]. S.A. Balbus and J.F. Hawley, Astrophys. J. 376, 214-222 (1991), doi:10.1086/170270

[26]. C. Nipoti and L. Posti, e-print arXiv:1206.3890v2 [astro-ph.GA] (2012).

[27]. V.P. Lakhin and V.I. Ilgisonis, On the Influence of Dissipative Effects on Instabilities of Differentially-Rotating Plasmas, JETP. 137(4), 783-788 (2010). (in Russian)

[28]. O.N. Kirillov and F. Stefani, Proceedings of the International Astronomical Union. 8, 233-234 (2012), doi: $10.1017 /$ S1743921312019771.

[29]. O.N. Kirillov, F. Stefani and Y. Fukumoto, J. Fluid Mech. 760, 591- 633 (2014), doi:10.1017/jfm.2014.614

[30]. G. Rüdiger, R. Hollerbach and L.L. Kitchatinov, Magnetic Processes in Astrophysics: Theory, Simulations, Experiment.(Wiley-VCH, 2013). p.356, doi:10.1002/9783527648924.

[31]. A.M. Soward, Phys. Earth Planet Int. 20(2-4), 134-151 (1979), doi:10.1016/0031-9201(79)90036-0.

[32]. S. Childress and A.M. Soward, Phys. Rev. Lett. 29, 837-839 (1972), doi:10.1103/PhysRevLett.29.837.

[33]. F.H. Busse, Phys. Earth Planet. Int. 12,. 350-358 (1976), doi:10.1016/0031-9201(76)90030-3.

[34]. F. Busse and F. Finocchi, Physics of The Earth and Planetary Interiors. 80(1-2), 13-23 (1993), doi:10.1016/00319201(93)90069-L.

[35]. E. Kurt, F.H. Busse and W. Pesch, Theoret. Comput. Fluid Dynamics, 18, 251-263 (2004), doi:10.1007/s00162-004-0132-6.

[36]. M.I. Kopp, A.V. Tur and V.V. Yanovsky, Problems of Atomic Science and Technology, 4(116), 230-234 (2018), arXiv: $1805.11894 \mathrm{v} 1[$ astro-ph.EP](2018).

[37]. V.P. Maslov and M.V. Fedoriuk, Квазиклассическое приближение для уравнений квантовой механики [Quasi-Classical Approximation for Quantum Mechanics Equations], (Nauka, Moscow, 1976), p. 296. (in Russian)

[38]. A.B. Mikhailovskii, Теория плазменных неустоичивостей. T.2. Неустойчивости неоднородной плазмы [Theory of Plasта Instabilities V.2, Inhomogeneous plasma instabilities], (Atomizdat, Moscow, 1971). p. 312. (in Russian)

[39]. D.A. Shalybkov, Гидродинамическая и гидромагнитная устойчивость течения Куэтта [Hуdrodynamic and hydromagnetic stability of the Couette flow], Usp. Fiz. Nauk, 179(9), 971-993 (2009), doi:10.3367/UFNe.0179.200909d.0971. (in Russian)

[40]. R. Narayan, E. Quataert, I.V. Igumenshchev and M.A. Abramowicz, Astrophys. J. 577, 295-301 (2002).

[41]. H. Ji, J. Goodman and A. Kageyama, Mon. Not. R. Astron. Soc. 325, L1-L5 (2001), doi:10.1046/j.1365-8711.2001.04647.x.

[42]. K. Noguchi, V.I. Pariev, S.A. Colgate, H.F Beckley and J. Nordhaus, Astrophys. J. 575, 1151-1162 (2002).

[43]. E.P. Velikhov, A.A. Ivanov, V.P. Lakhin, and K.S Serebrennikov, Phys. Letters A. 356, 357-365 (2006), doi:10.1016/j.physleta.2006.03.073.

[44]. F.R. Gantmakher, Лекции по аналитической механике [Lectures in analytical mechanics], (Fizmatlit, Moscow, 2005), p. 264. (in Russian)

[45]. Moffat G. Возбуждение магнитного поля в проводящей среде [Magnetic Field Generation in Electrically Conducting Fluids], (Mir, Moscow, 1980), p. 343p. (in Russian)

[46]. G. Rudiger and M. Kuker, arXiv:1601.03877v1[astro-ph.SR] (2016).

[47]. F. Krauze and K.H. Redler, Магнитная гидродинамика средних полей и теория динамо [Mеап-Field Magnetohydrodynamics and Dynamo Theory], (Mir, Moscow, 1984), p. 314p. (in Russian)

[48]. A.V. Getling, Astronomy Reports, 45, 569-576 (2001), doi:10.1134/1.1383816.

[49]. A.V. Getling, Astronomy Reports, 56, 395-402 (2012), doi:10.1134/S106377291.

[50]. A.V. Getling, Solar Physics, 239, 93-111 (2006), doi:10.1007/s11207-006-0231-1.

[51]. P. Goldreich and D. Lynden-Bell, Mon. Not. R. Astron. Soc. 130(2), 125-158 (1965), doi:10.1093/mnras/130.2.125.

[52]. E. Knobloch and K. Jullien, Physics of Fluids, 17(9), 094106 (2005), doi:10.1063/1.2047592.

[53]. R. Haberman, Elementary Applied Partial Differential Equations with Fourier Series and Boundary Value Problems, 4th ed. (Pearson/Prentice Hall, N.J., 2004), p. 769.

[54]. P.G. Siddheshwar and C. Kanchana, Int. J. Mech. Sci. 131, 1061-1072 (2017), doi:10.1016/j.ijmecsci.2017.07.050. 